\title{
A Mission-Adaptive Variable Camber Flap Control System to Optimize High Lift and Cruise Lift-to-Drag Ratios of Future N+3 Transport Aircraft
}

\author{
James Urnes, Sr. * \\ Boeing Research \& Technology, St. Louis, MO 63134 \\ Nhan Nguyen $†$ Corey Ippolito $¥$ Joseph Totah ${ }^{\S}$ \\ NASA Ames Research Center, Moffett Field, CA 94035 \\ Khanh Trinh ", Eric Ting $\|$ \\ Stinger Ghaffarian Technologies, Inc. / NASA Ames Research Center, Moffett Field, CA 94035
}

\begin{abstract}
Boeing and NASA are conducting a joint study program to design a wing flap system that will provide mission-adaptive lift and drag performance for future transport aircraft having light-weight, flexible wings. This Variable Camber Continuous Trailing Edge Flap (VCCTEF) system offers a lighter-weight lift control system having two performance objectives: (1) an efficient high lift capability for take-off and landing, and (2) reduction in cruise drag through control of the twist shape of the flexible wing. This control system during cruise will command varying flap settings along the span of the wing in order to establish an optimum wing twist for the current gross weight and cruise flight condition, and continue to change the wing twist as the aircraft changes gross weight and cruise conditions for each mission segment. Design weight of the flap control system is being minimized through use of light-weight shape memory alloy (SMA) actuation augmented with electric actuators. The VCCTEF program is developing better lift and drag performance of flexible wing transports with the further benefits of lighter-weight actuation and less drag using the variable camber shape of the flap.
\end{abstract}

\section{Introduction}

The aircraft industry has been responding to the need for energy-efficient aircraft by redesigning airframes to be aerodynamically efficient, employing light-weight materials for aircraft structures and incorporating more energyefficient aircraft engines. Reducing airframe operational empty weight (OEW) using advanced composite materials is one of the major considerations for improving energy efficiency. Modern light-weight materials can provide less structural rigidity while maintaining sufficient load-carrying capacity. As structural flexibility increases, aeroelastic interactions with aerodynamic forces and moments can alter aircraft aerodynamics significantly, thereby potentially degrading aerodynamic efficiency.

Under the Fundamental Aeronautics Program at the NASA Aeronautics Research Mission Directorate, the Fixed Wing (FW) project is conducting discipline-based and multidisciplinary foundational research to investigate advanced concepts and technologies for future aircraft systems.

A NASA study entitled "Elastically Shape Future Air Vehicle Concept" was conducted in $2010^{1}$ to examine new concepts that can enable active control of wing aeroelasticity to achieve drag reduction. This study showed that highly flexible wing aerodynamic surfaces can be elastically shaped in-flight by active control of wing twist and vertical deflection in order to optimize the local angle of attack of wing sections to improve aerodynamic efficiency through drag reduction during cruise and enhanced lift performance during take-off and landing.

\footnotetext{
*Program Manager, Platform Systems/Subsystems Technology, james.m.urnes-sr@ boeing.com, (314)234-3775

†Associate Fellow AIAA, Intelligent Systems Division, nhan.t.nguyen@nasa.gov, (650)604-4063

${ }^{\ddagger}$ Intelligent Systems Division, corey.a.ippolito@nasa.gov, (650)604-1605

§Associate Fellow AIAA, Intelligent Systems Division, joseph.j.totah@nasa.gov, (650)604-1864

II Intelligent Systems Division, khanh.v.trinh@nasa.gov, (650)604-5280

"Intelligent Systems Division, eric.b.ting@ nasa.gov
} 
The study shows that active aeroelastic wing shaping control can have a potential drag reduction benefit. Conventional flap and slat devices inherently generate drag as they increase lift. The study shows that conventional flap and slat systems are not aerodynamically efficient for use in active aeroelastic wing shaping control for drag reduction. A new flap concept, referred to as Variable Camber Continuous Trailing Edge Flap (VCCTEF) system, was developed to address this need. Initial results indicate that the VCCTEF system may offer a potential pay-off for drag reduction that will result in significant fuel savings. In order to realize the potential benefit of drag reduction by active aeroelastic wing shaping control, configuration changes in high-lift devices have to be a part of the wing shaping control strategy.

A second study was awarded in 2011 under the NASA Subsonic Fixed Wing (SFW) project, entitled "Development of Variable Camber Continuous Trailing Edge Flap System". ${ }^{2}$ This study built upon the development of the VCCTEF system for NASA Generic Transport Model (GTM) which is essentially based on the B757 airframe, ${ }^{3}$ employing light-weight shaped memory alloy (SMA) technology for actuation and three separate chordwise segments shaped to provide a variable camber to the flap. This cambered flap has potential for drag reduction as compared to a conventional straight, plain flap. The flap is also made up of individual 2-foot spanwise sections which enable different flap setting at each flap spanwise position. This results in the ability to control the wing twist shape as a function of span, resulting in a change to the wing twist to establish the best lift-to-drag ratio (L/D) at any aircraft gross weight or mission segment. Current wing twist on commercial transports is permanently set for one cruise configuration, usually for a $50 \%$ loading or mid-point on the gross weight schedule. The VCCTEF offers different wing twist settings for each gross weight condition and also different settings for climb, cruise and descent, a major factor in obtaining best L/D conditions.

The second feature of VCCTEF is a continuous trailing edge flap. The individual 2-foot spanwise flap sections are connected with a flexible covering, so no breaks can occur in the flap platforms, thus reducing drag by eliminating these breaks in the flap continuity which otherwise would generate vorticity that results in a drag increase and also contributes to airframe noise. This continuous trailing edge flap design combined with the flap camber result in lower drag increase during flap deflections. In addition, it also offers a potential noise reduction benefit.

The VCCTEF project has four objectives:

1. Use the VCCTEF to twist the flexible wing to obtain changes in lift-to-drag ratios that will reduce cruise drag throughout the flight envelope.

2. Determine aeroelastic control approaches that will compensate for reduced flutter margins of flexible wings.

3. Use the full span cambered flap as the primary high lift device for take-off, climb-out, let-down and final approach.

4. Use the aft section of the cambered flap as a roll control effector and as an aeroservoelastic control device of flexible wing structure dynamic modes.

\section{Performance Benefit}

Drag reduction by increasing lift-to-drag ratios through reshaping the wing twist has a significant advantage if the wing has structural flexibility properties that will permit this change in shape. Current transport wing design incorporates a twist profile that provides efficient lift-to-drag ratios, and the twist angle is set for an average flight profile and at the mid-point gross weight. Figure 1 shows results of drag polars of a flexible wing transport for three fuel loadings: $80 \%$ fuel load early in cruise, $50 \%$ fuel load at the mid-point cruise, and $20 \%$ fuel load toward the end of cruise.

The 1-g trim points show the lowest drag at the 50\% mid-point that is indicative of the wing twist design, with a significant drag increase at the other fuel loading conditions due to wing shape changes for a flexible wing transport.

Using the VCCTEF, the drag polars can be changed to reduce drag, two examples are shown in Figure $1 .{ }^{1}$ The important factor is to maintain the desired wing twist through the deflection of the VCCTEF, but not to lose the drag benefit by having too much increase in drag due to the flap deflections. Hence, the use of the variable camber flap instead of conventional flaps can offer a better drag signature. 


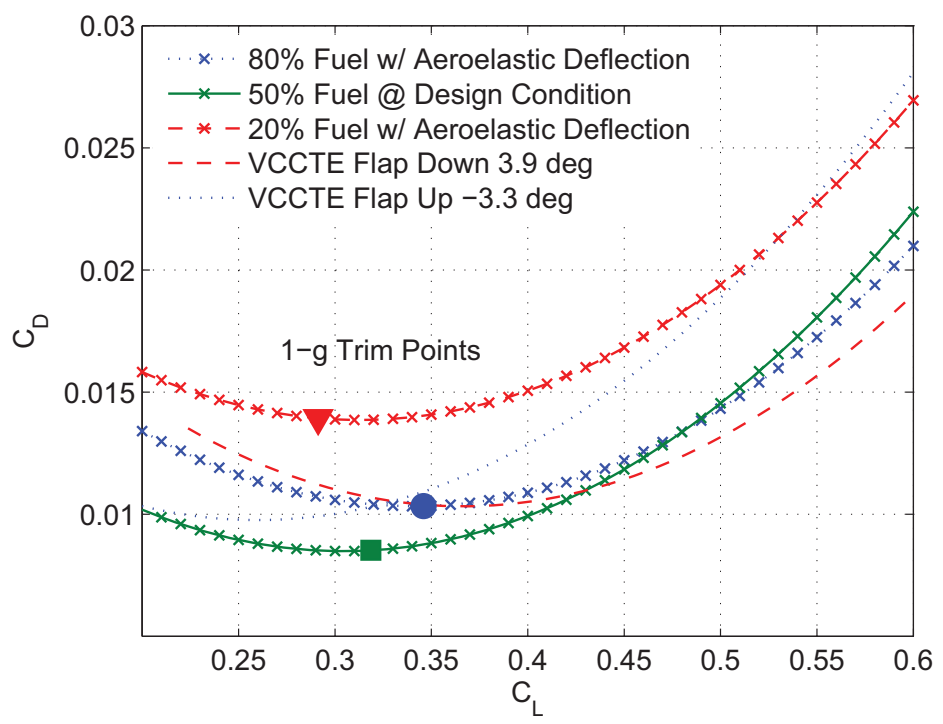

Nguyen, N., “Elastically Shaped Future Air Vehicle Concept,” NASA Innovation Fund Award 2010 Report, October 2010

Submitted to NASA Innovative Partnerships Program

Figure 1. Wing Shaping Control can Change the Lift-to-Drag Ratio to Minimize Trim Drag

\section{Design of the VCCTEF System}

Figure 2 shows a wing and flap layout of the VCCTEF design for the GTM.

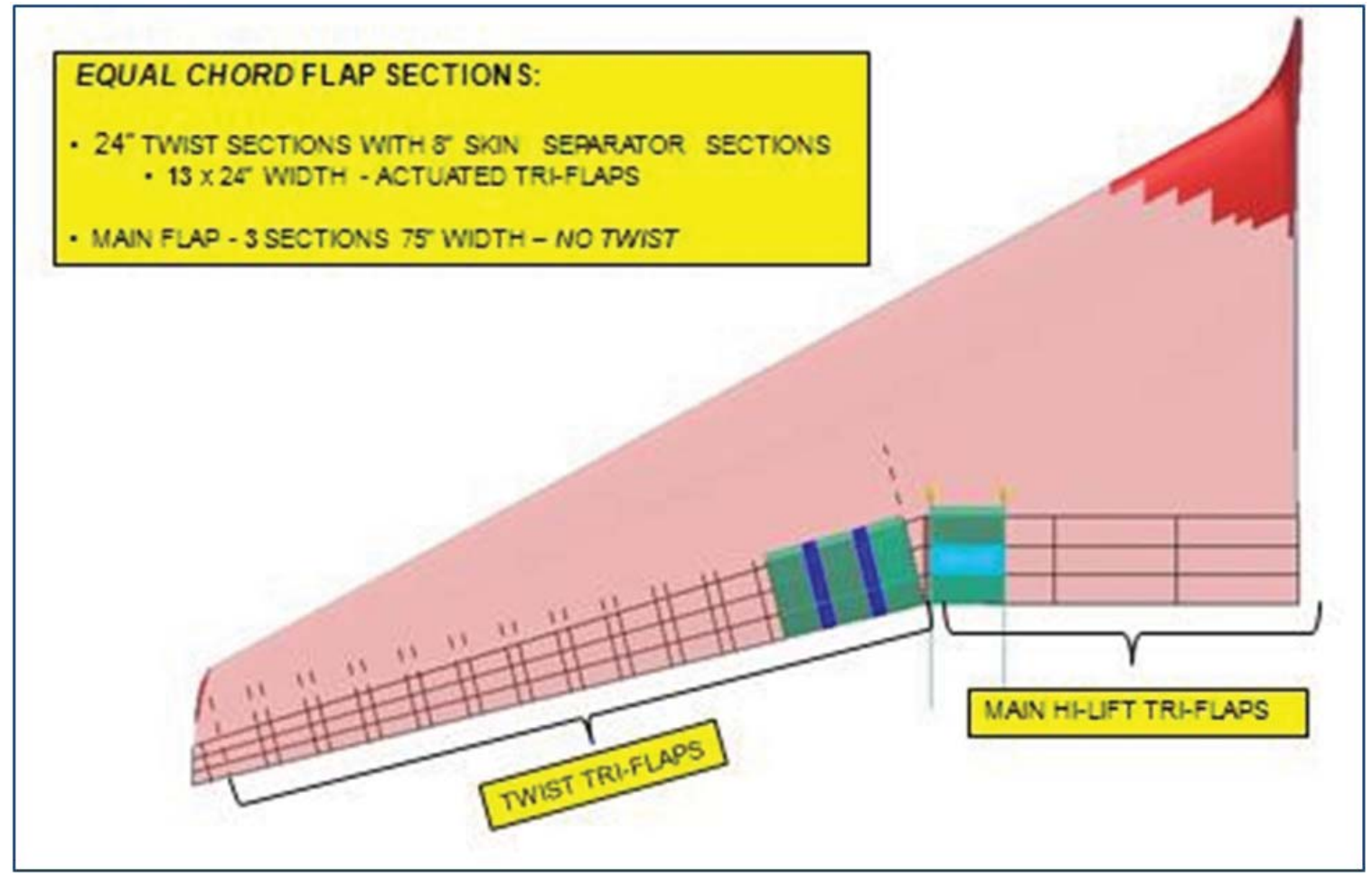

Figure 2. Wing Configured with the Variable Camber Continuous Trailing Edge Flap

The flap is divided into 14 sections attached to the outer wing and 3 sections attached to the inner wing. Each 24-inch section has three camber flap segments that can be individually commanded. These camber flaps are joined to 
the next section by a flexible and supported material (shown in blue) installed with the same shape as the camber and thus providing continuous flaps throughout the wing span with no drag producing gaps.

A major goal of the program is to develop a light-weight flap control system that has a significant weight advantage as compared to current flap screw-jack actuators. Hydraulic, electric and Shape Memory Alloy (SMA) torque rod actuation were evaluated with the result that the SMA actuation has the best weight advantage, as shown in Figure 3 hardware comparisons.

Moreover, the use of hinge line actuation eliminates the large and heavy external mounted actuators, and permits all actuators to be interior to the wing and flap mold lines, thus contributing to the overall drag reduction goal.

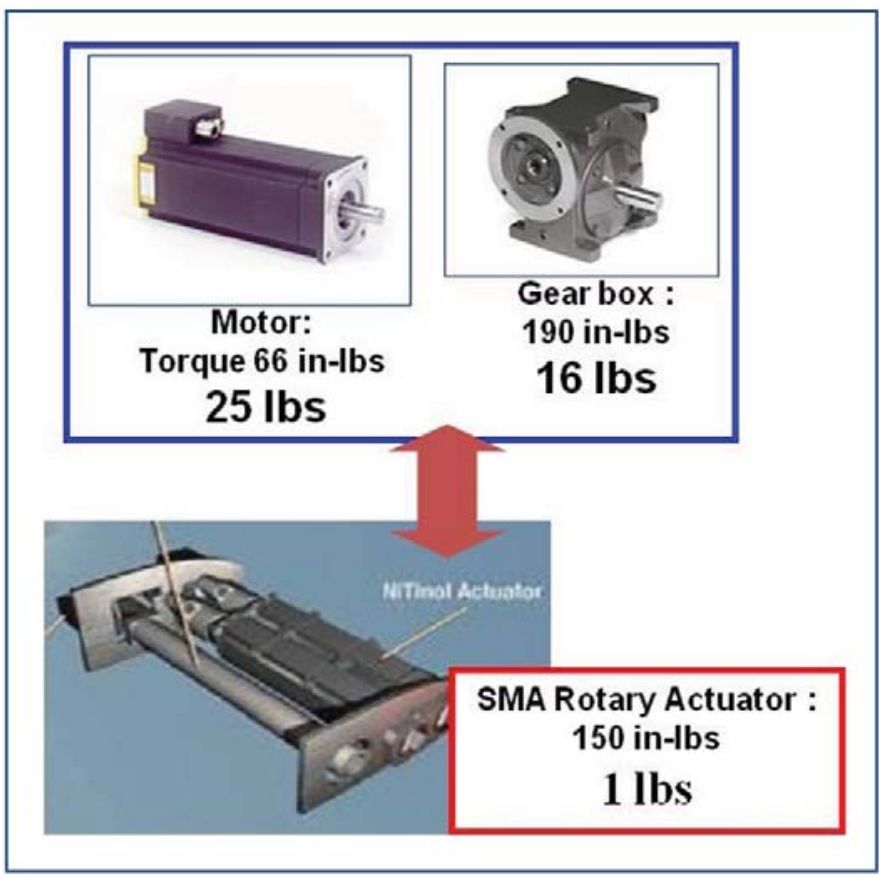

Figure 3. Shape Memory Alloy Actuators Have a Low Weight Compared to Electric Motor Actuators

Figure 4 shows three of the outer wing flap sections, each having three camber components.

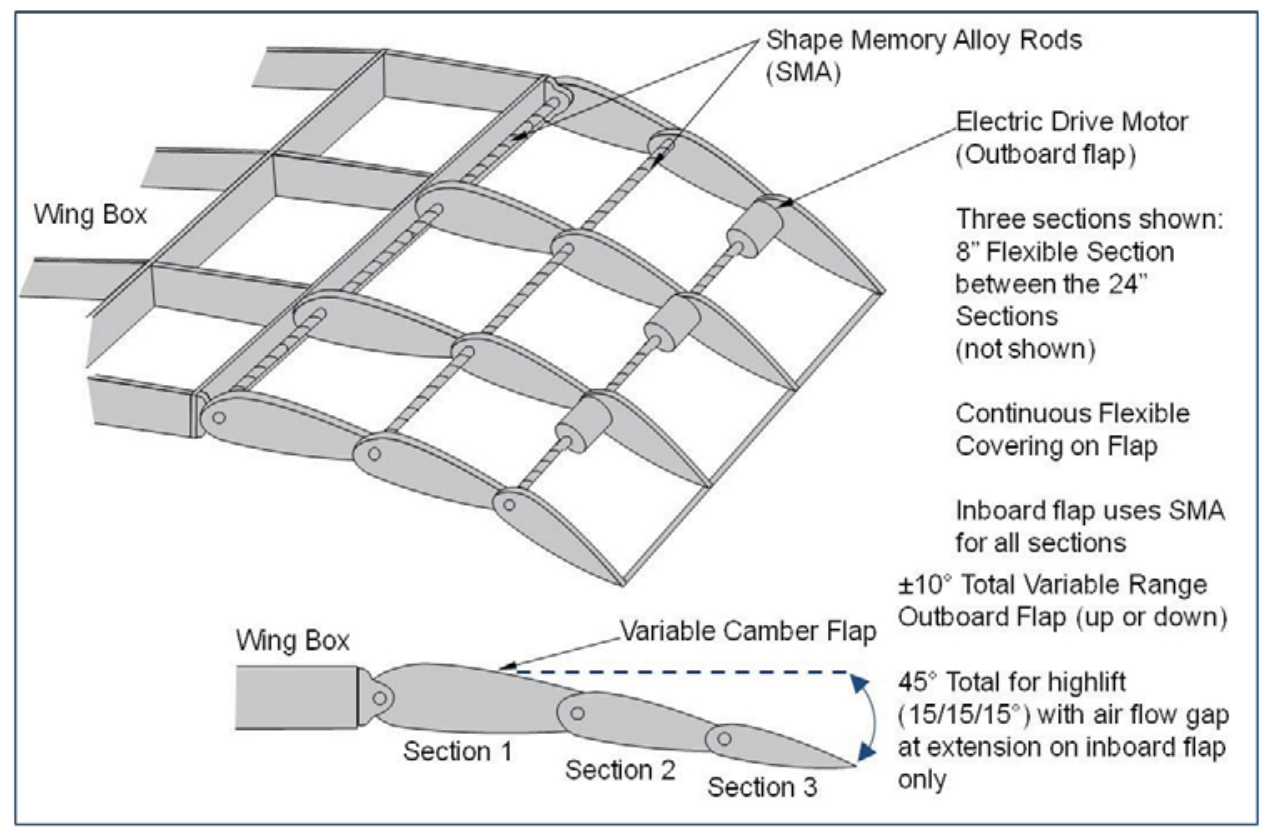

Figure 4. The Variable Camber Flap Control uses Shape Memory Alloy Torque Rod and Electric Drive Actuation 
SMA actuators drive the first and second camber flap segments and a faster acting electric actuator drives the third camber flap segment. SMA actuators can deliver large hinge moments, but generally move at a slow rate. The outer wing flap uses the full-span third camber segment as a roll command effector and as a control device for suppressing aeroelastic wing structural dynamic modes, both requiring high rates which can be met by electric actuators. Figure 5 shows an SMA actuator.

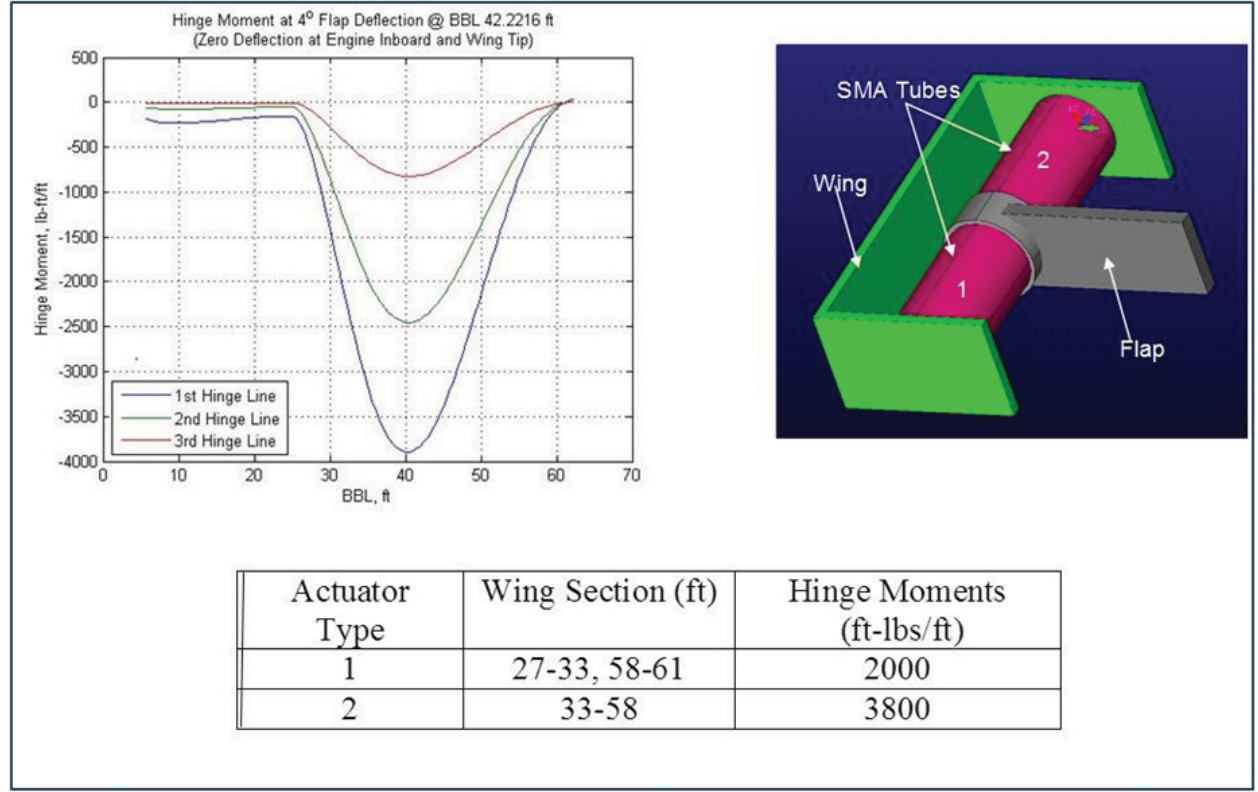

Figure 5. Shape Memory Alloy Actuators can Meet the VCCTEF Hinge Moment Requirements

\section{High Lift Configuration}

Using the camber positioning, a full-span, low-drag, high-lift configuration can be activated that has no drag producing gaps and a low flap noise signature. This is shown in Figure 6.

To further augment lift, a slotted flap configuration is formed by an air passage between the wing and the inner flap that serves to improve airflow over the flap and keep the flow attached. This air passage appears only when the flaps are extended in the high lift configuration.

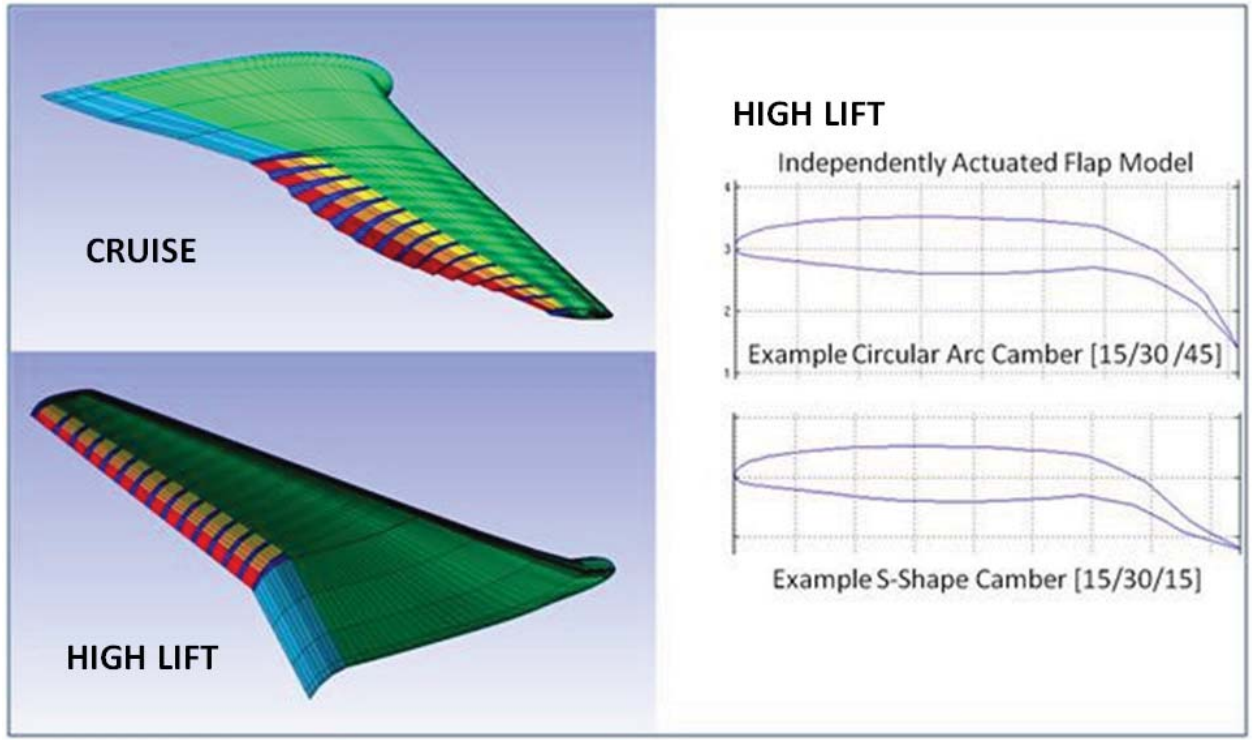

Figure 6. Cruise and High Lift VCCTEF Configurations 
In the high-lift configuration, the outer wing flap uses the third camber section for roll control, as shown in Figure 6. This provides rolling moment that is equivalent to aileron control. It is somewhat similar to deflecting the ailerons in a droop position to act as flaps, a common procedure used on tactical aircraft and on some transport aircraft.

The high-lift configuration distributes the required flap hinge moment throughout the span of the wing while using actuation components that are all located interior to the wing and flap. This can be achieved by the use of SMA hinge line torque rods, sized to meet the hinge moment requirements at each spanwise location on the wing. This distribution of actuator load permits efficient sizing of the actuators, and eliminates the need for large external flap actuators and associated drag from these installations.

\section{Vortex-Lattice Flexible Aircraft Geometry Modeling}

The GTM configured with the VCCTEF is analyzed using NASA vortex-lattice code Vorview. ${ }^{4}$ This code is also used to optimize lift-to-drag ratios of the VCCTEF wings. Vorview reads in aircraft geometries, flight states including altitude, airspeed, angle of attack and aircraft weight and computes aircraft aerodynamic coefficients, stability and control derivatives, and distributions of wing lift, drag, and pitching moment coefficients. The aircraft geometry in Vorview is defined by a surface mesh. The optimization process involved running multiple Vorview analyses comparing different flap settings of the VCCTEF. In the optimization process, a new mesh model was produced for each new flap setting on the fly generated by an automated geometry generation tool developed in Matlab.

Figure 7 shows a mesh model of the GTM with the VCCTEF. The mesh model contains 6 components: fuselage, wings, engines, pylons, horizontal tails and vertical tails. Each component is a structured mesh containing quadrilateral elements defining the outer mold line of the component geometry. Each component is treated as a cylinder topologically. Non-uniform section cuts were placed along cylinder axes and non-uniform mesh nodes were placed along the circumferential direction of each section cuts to capture the aircraft geometry while keeping the number of elements down. Each component was meshed separately. Vorview does not require a conformal mesh at intersections of intersecting components. However, Vorview does require the geometry to be water-tight. To produce a water-tight mesh, intersecting ends of wings, pylon, horizontal tails and vertical tails were extended so that the end nodes protruded into adjacent components. The aircraft mesh has a total of 20,806 nodes and 19,908 quadrilateral elements. Table 1 shows mesh size data for the 6 components.

\begin{tabular}{|c|c|c|c|}
\hline & Number of Sections & Number of Nodes per Section & Number of Quad Elements \\
\hline \hline Fuselage & 84 & 101 & 8300 \\
\hline Wings & 80 & 115 & 8892 \\
\hline Engines & 32 & 21 & 600 \\
\hline Pylons & 4 & 23 & 44 \\
\hline Horizontal Tails & 18 & 81 & 1280 \\
\hline Vertical Tail & 20 & 45 & 792 \\
\hline
\end{tabular}

Table 1. Mesh Size Data of Geometry Model

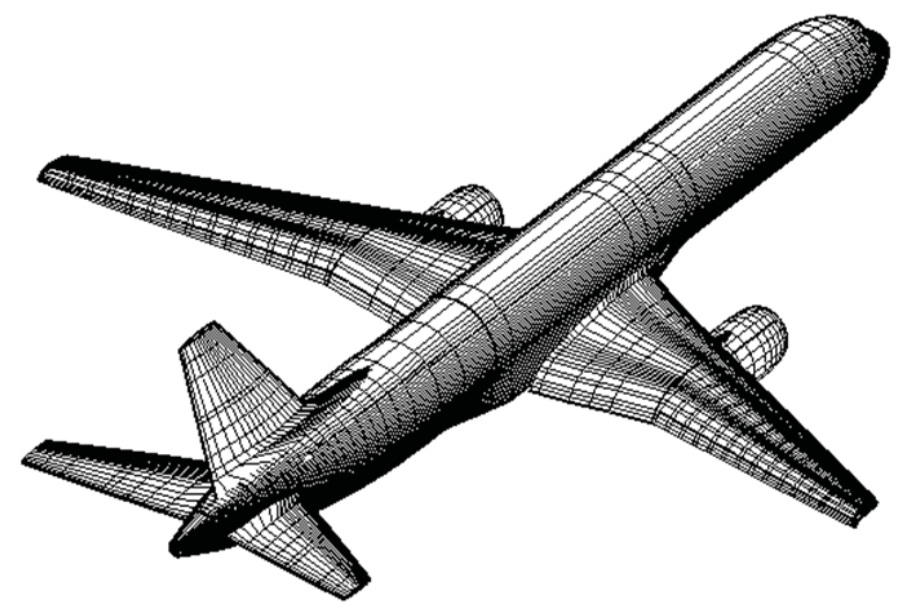

Figure 7. Vorview GTM Mesh 
In meshing the VCCTEF wings, section cuts were placed at every flap/follower flexible material boundary. This allows the mesh to correctly define the VCCTEF geometry in the wing spanwise direction. Likewise, mesh nodes along each wing sections were placed to correctly define the flap geometry in the wing chord-wise direction. There were 3 quads along the chord-wise direction of each of the 3 chordwise flap sections. For each flap setting, the locations of the 3 flap hinge rods were determined first. Then node locations of the quads defining each flap were calculated. Thus, the sides of the flap quads follow the flap geometry boundaries. Figure 8 shows mesh of VCCTEF wings and mesh sections for 2 different flap settings.

Vorview processes aircraft quad surface mesh along with user-defined control parameters to define slices and sub polygons that are subsequently used in the aerodynamic calculations. Figure 8 shows Vorview slices and sub-polygons of the GTM with VCCTEF, respectively.

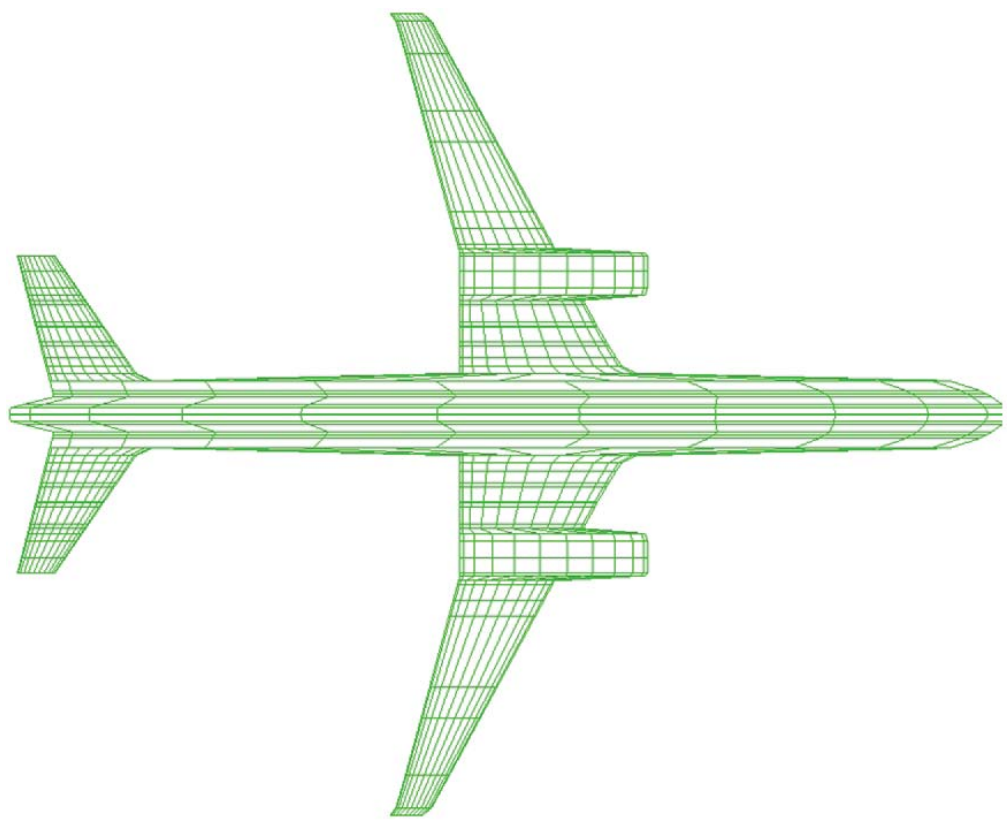

Figure 8. Vorview GTM Sub-Polygons

The variable camber flap is modeled with three chordwise flap segments as shown in Figure 9. ${ }^{1}$

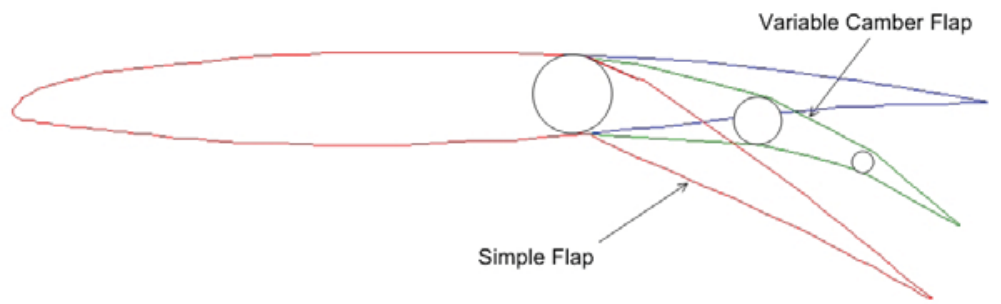

Nguyen, N., "Elastically Shaped Future Air Vehicle Concept," NASA Innovation Fund Award 2010 Report, October 2010

Submitted to NASA Innovative Partnerships Program

Figure 9. Variable Camber Flap

The variable camber flap geometry is specified by three deflection values $\delta_{f_{1}}, \delta_{f_{2}}$, and $\delta_{f_{3}}$ for the innermost, intermediate, and outermost flap segments, respectively. The baseline camber shape is described by a circular arc, with each flap segment deflected by the same amount relative to each other. With a circular arc camber, only one flap deflection command is needed. For example, for a commanded flap deflection of $12^{\circ}$, the innermost flap segment is deflected $4^{\circ}$, the intermediate flap segment is deflected $8^{\circ}$, and the outermost flap segment is deflected by $12^{\circ}$. Thus, in general

$$
\delta_{f_{i}}=\frac{i \delta_{f_{c}}}{3}
$$

$$
7 \text { of } 24
$$


where $i=1,2,3$, and $\delta_{f_{c}}$ is the commanded flap deflection.

The camber angle of the circular arc camber flap is the difference between between $\delta_{f_{3}}$ and $\delta_{f_{1}}$. Thus, the variable camber angle $\chi=2 \delta_{f_{c}} / 3$ is a function of the commanded flap deflection. A camber flap is more effective in producing lift than a straight uncambered flap. As the camber increases, the pressure distribution at the aft of the airfoil increases that results in a lift increase.

The variable camber flap produces about the same downwash as a simple plain flap deflected by the same angle as shown in Figure 9-. However, the normal surface area of the variable camber flap exposed to the flow field is significantly reduced. Thus, the drag reduction benefit of the variable camber flap is realized since the pressure drag across the flap surface is reduced due to less exposed normal surface area.

In order to model a flexible wing aircraft, an automated geometry generation tool is developed in Matlab. This geometry modeling uses structural deflection data computed by a finite-element model (FEM) to update the undeformed aircraft wing geometry to reflect static aeroelastic deflections. The deformed geometry, which reflects a series of coordinate transformations on the outer mold line of the jig-shape aircraft wing, is processed into a geometry file that can be used directly in vortex-lattice calculations.

With reference to Figure 10, the coordinate reference frame $\left(x_{B}, y_{B}, z_{B}\right)$ defines the Body Station (BS), the Body Butt Line $(\mathrm{BBL})$, and the Body Water Line (BWL) of the aircraft, respectively. The coordinate reference frame $\left(x_{V}, y_{V}, z_{V}\right)$ is the translated coordinate system attached to the nose of the aircraft such that $x_{V}=x_{B}-13.25 \mathrm{ft}, y_{V}=y_{B}$, and $z_{V}=z_{B}-15.8333 \mathrm{ft}$. This reference frame is used for the vortex-lattice aerodynamic modeling and optimization. The stability reference frame $(x, y, z)$ is attached to the CG such that $x=\bar{x}_{V}-x_{V}, y=y_{V}-\bar{y}_{V}$, and $z=\bar{z}_{V}-z_{V}$, where $\left(\bar{x}_{V}, \bar{y}_{V}, \bar{z}_{V}\right)$ is the coordinate of the CG in the $\left(x_{V}, y_{V}, z_{V}\right)$ reference frame. ${ }^{5}$

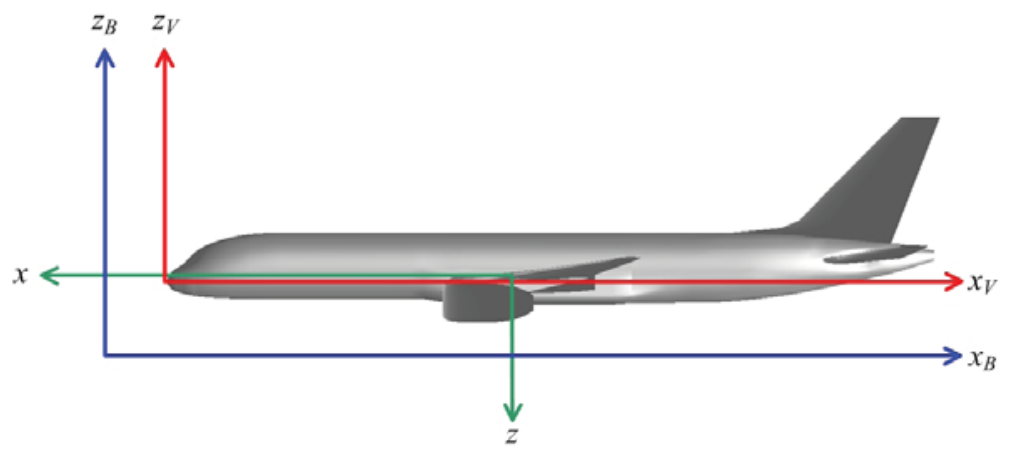

Figure 10. GTM Coordinate Systems

The wing reference frame is defined by the coordinate reference frame $\left(x_{W}, y_{W}, z_{W}\right)$ as shown in Figure 11 . The $x_{W}$-axis is the wing elastic axis, the $y_{W}$-axis points aft along the chord direction, and the $z_{W}$-axis is the wing normal direction.

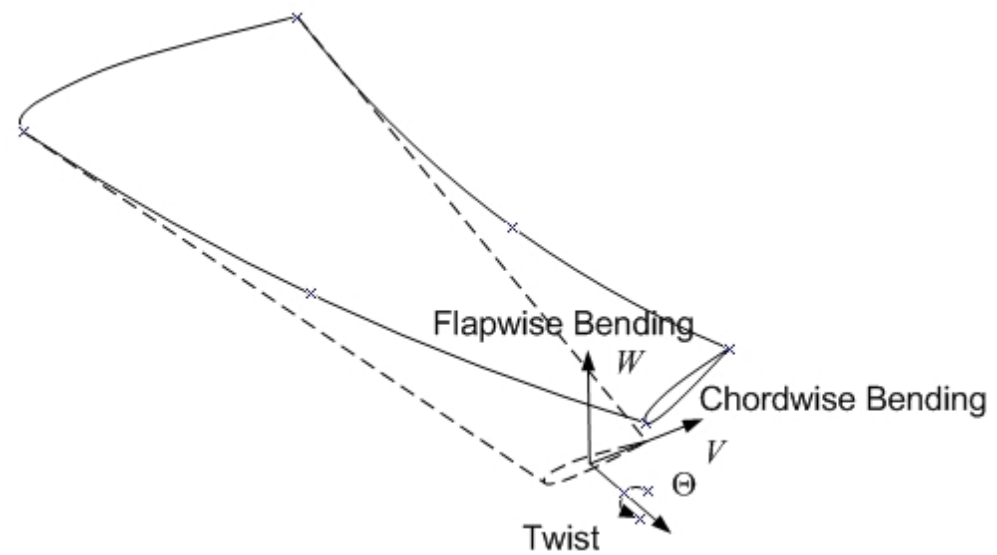

Figure 11. Wing Bending Deflections and Twist 
Neglecting chordwise bending deflection, the aeroelastic deflections in bending and torsion are expressed in a vector form as

$$
\begin{gathered}
\phi=\Theta \mathbf{i}_{W}-W_{x} \mathbf{j}_{W} \\
\delta=-W \sin W_{x} \mathbf{i}_{W}+W \cos W_{x} \mathbf{k}_{W}
\end{gathered}
$$

where $\Theta$ is the wing twist (positive nose-down), $W_{x}$ is the wing bending slope (positive slope upward), and $\left(\mathbf{i}_{W}, \mathbf{j}_{W}, \mathbf{k}_{W}\right)$ are the unit vectors corresponding to $\left(x_{W}, y_{W}, z_{W}\right)$.

The coordinate reference frame $\left(x_{W}, y_{W}, z_{W}\right)$ is related to the coordinate reference frame $\left(x_{V}, y_{V}, z_{V}\right)$ by the following relationship:

$$
\left[\begin{array}{l}
\mathbf{i}_{W} \\
\mathbf{j}_{W} \\
\mathbf{k}_{W}
\end{array}\right]=\left[\begin{array}{ccc}
-\sin \Lambda \cos \Gamma & -\cos \Lambda \cos \Gamma & -\sin \Gamma \\
-\cos \Lambda & \sin \Lambda & 0 \\
\sin \Lambda \sin \Gamma & \cos \Lambda \sin \Gamma & -\cos \Gamma
\end{array}\right]\left[\begin{array}{c}
-\mathbf{i}_{V} \\
-\mathbf{j}_{V} \\
-\mathbf{k}_{V}
\end{array}\right]
$$

where $\Lambda$ is the sweep axis of the elastic axis, $\Gamma$ the wing dihedral angle, and $-\left(\mathbf{i}_{V}, \mathbf{j}_{V}, \mathbf{k}_{V}\right)$ are the unit vectors corresponding to $\left(x_{V}, y_{V}, z_{V}\right)$.

Thus, the aeroelastic deflections result in a wing twist expressed as an incremental angle of attack $\Delta \alpha$ (positive nose-up), a horizontal deflection $\Delta y_{V}$ (positive displacement toward wing tip), and a vertical deflection $\Delta z_{V}$ (positive displacement upward) as follows:

$$
\begin{gathered}
\Delta \alpha=-\Theta \cos \Lambda \cos \Gamma-W_{x} \sin \Lambda \\
\Delta y_{V}=-W \sin W_{x} \cos \Lambda \cos \Gamma-W \cos W_{x} \cos \Lambda \sin \Gamma \\
\Delta z_{V}=-W \sin W_{x} \sin \Gamma+W \cos W_{x} \cos \Gamma
\end{gathered}
$$

A coordinate transformation to account for wing aeroelastic deflections is performed by rotating a wing section about its elastic axis by the incremental angle of attack $\Delta \alpha$ and then translating the resultant coordinates by the horizontal deflection $\Delta y_{V}$ and the vertical deflection $\Delta z_{V}$ according to

$$
\begin{gathered}
x_{V}^{\prime}=x_{e a}+\left(x_{V}-x_{e a}\right) \cos \Delta \alpha-\left(z_{V}-z_{e a}\right) \sin \Delta \alpha \\
y_{V}^{\prime}=y_{V}+\Delta y_{v} \\
z_{V}^{\prime}=z_{e a}+\Delta z_{V}+\left(x_{V}-x_{e a}\right) \sin \Delta \alpha+\left(z_{V}-z_{e a}\right) \cos \Delta \alpha
\end{gathered}
$$

where the prime superscript denotes the transformed coordinates, and $\left(x_{e a}, y_{e a}, z_{e a}\right)$ is the coordinate of the elastic axis in the reference frame $\left(x_{V}, y_{V}, z_{V}\right)$.

\section{Roll Control Analysis}

An investigation of the roll control authority offered by the VCCTEF is conducted. As a concept, roll control using the VCCTEF is accomplished by utilizing solely the outermost camber flap segments of the 14 spanwise sections of the VCCTEF on the wing portion outboard from the engines. Vorview is employed with the flap model to analyze roll control derivatives. The initial analysis does not include the effect of aeroelastic deflections which generally decrease the roll control effectiveness.

In order to analyze the asymmetric deflection of the roll control segments of the VCCTEF, A vortex-lattice analysis is performed on three separate cases for each flight condition. First, a nominal baseline is established based on a trim deflection of the VCCTEF $\left(\delta_{f_{1}}, \delta_{f_{2}}, \delta_{f_{3}}\right)$ which represents the deflections of the innermost, intermediate, and outermost camber segments of a VCCTEF spanwise flap section. The second and third vortex-lattice cases are then computed for $\delta_{f_{3}} \pm \Delta \delta_{f_{3}}$ on the $14 \mathrm{VCCTEF}$ spanwise flap sections, with $\Delta \delta_{f_{3}}=1^{\mathrm{o}}$. These three cases thus provide the wing spanwise distributions of lift and drag.

To generate a positive roll about the roll axis of the aircraft, the outermost camber segments are differentially deflected such that $\Delta \delta_{f_{3}}>0$ on the left wing and $\Delta \delta_{f_{3}}<0$ negative on the right wing. Let $y$ be the spanwise location of a wing station along the aircraft pitch axis where positive $y$ is along the right wing wing and negative $y$ is along the left wing. Aerodynamic data for spanwise wing stations are then obtained from the vortex-lattice solutions as shown in Figure 12. 


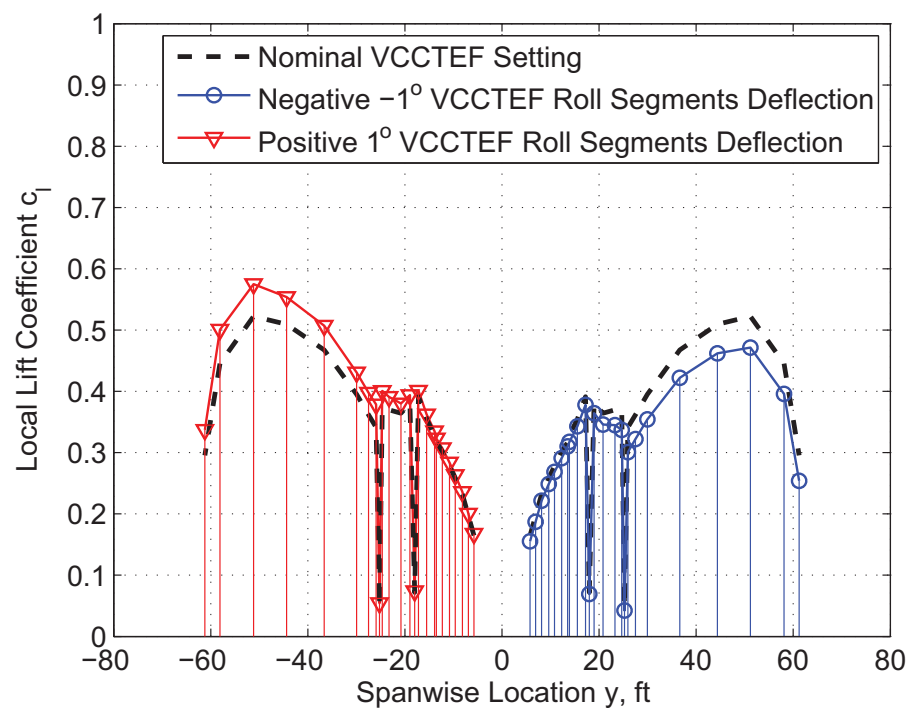

Figure 12. Local Lift Distribution with Differential Deflection of Outermost VCCTEF Segments for Roll Control

The spanwise lift and drag distributions on the left and right wings allow the rolling moment coefficient to be established in terms of the roll control derivative due to the deflection of the roll control segments of the VCCTEF.

$$
C_{l_{\delta_{f}}}=\frac{1}{S b \Delta \delta_{f_{3}}} \int_{-b / 2}^{b / 2}\left[\Delta c_{l}(y) \cos \alpha_{l}(y)-\Delta c_{d}(y) \sin \alpha_{l}(y)\right] c(y) y d y
$$

where $\Delta c_{l}$ and $\Delta c_{d}$ are the incremental section lift and drag coefficients, respectively, $c$ is the section chord, $S$ is the reference wing area, $b$ is the wing span, and $\alpha_{l}$ is the local angle attack defined as

$$
\alpha_{l}(y)=\alpha+\gamma(y)+\Delta \alpha(y)
$$

where $\gamma$ is the wing pre-twist distribution.

The magnitude of the drag contribution to the rolling moment is much smaller than that of the lift contribution and is not included in the estimate.

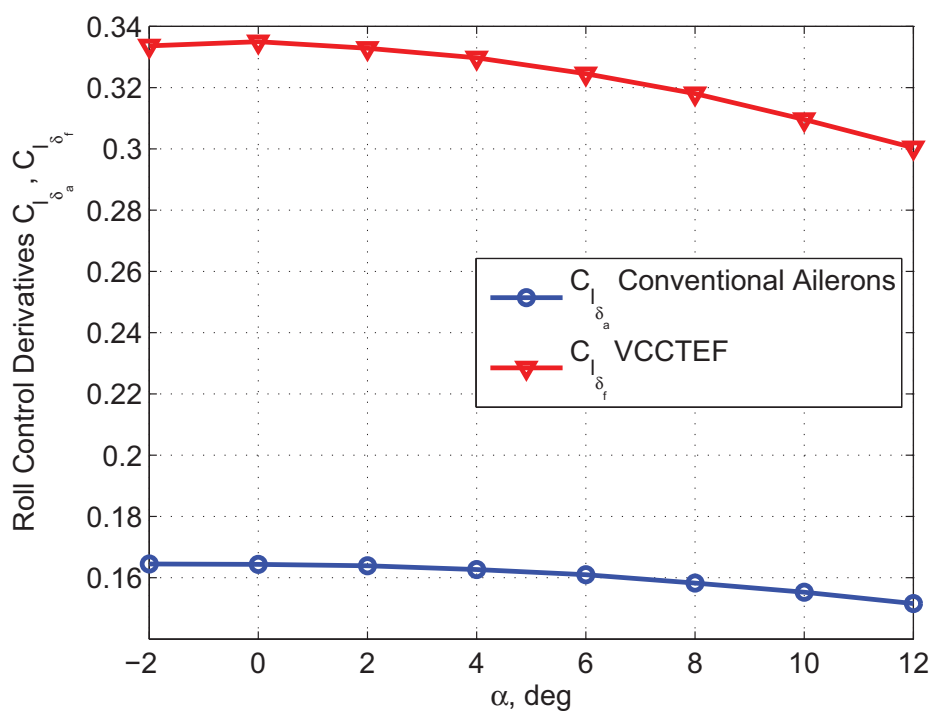

Figure 13. Comparison of Roll Control Derivatives due to VCCTEF $C_{l_{\delta_{f}}}$ and Conventional Ailerons $C_{l_{\delta_{a}}}$ at Cruise Conditions 
The values for $C_{l_{\delta_{f}}}$ due to the roll control segments of the VCCTEF are calculated for cruise conditions at Mach 0.8. These values are then compared to the values of the roll control derivative $C_{l_{\delta_{a}}}$ due to the conventional aileron system. ${ }^{5}$ The results in Figure 13 show that, at cruise conditions, the differential deflection of the roll control segments of the VCCTEF provides larger roll control derivatives than the conventional ailerons.

A roll control analysis at take-off is conducted assuming the VCCTEF is also being employed in a high-lift configuration. A conservative $C_{L_{\max }}$ value of 2.1098 with an additional $\Delta C_{L}=0.1$ due to ground effect is estimated without a lift augmentation due to the slotted flap configuration. This corresponds to the VCCTEF flap settings of $\left(\delta_{f_{1}}=15^{\circ}, \delta_{f_{2}}=30^{\circ}, \delta_{f_{3}}=45^{\circ}\right)$. The stall speed corresponding to this $C_{L_{\max }}$ value is Mach 0.1688 . Assuming conservatively that the lift-off speed $V_{\text {LOFF }}$ after rotation is $16 \%$ (normally $10 \%$ ) higher than $V_{\text {stall }}$, then a lift-off speed of Mach 0.196 is estimated.

However, because it is assumed that the VCCTEF will be retracting from the flap position limit at $45^{\circ}$ as the aircraft accelerates from lift-off, it is of interest to determine how much flap deflection is needed to provide a roll control authority similar to that of a conventional aileron system. Since maneuvering should not occur until after the plane has accelerated after lift-off, an airspeed of 150 knots (Mach 0.228 at sea-level) is used as an estimate.

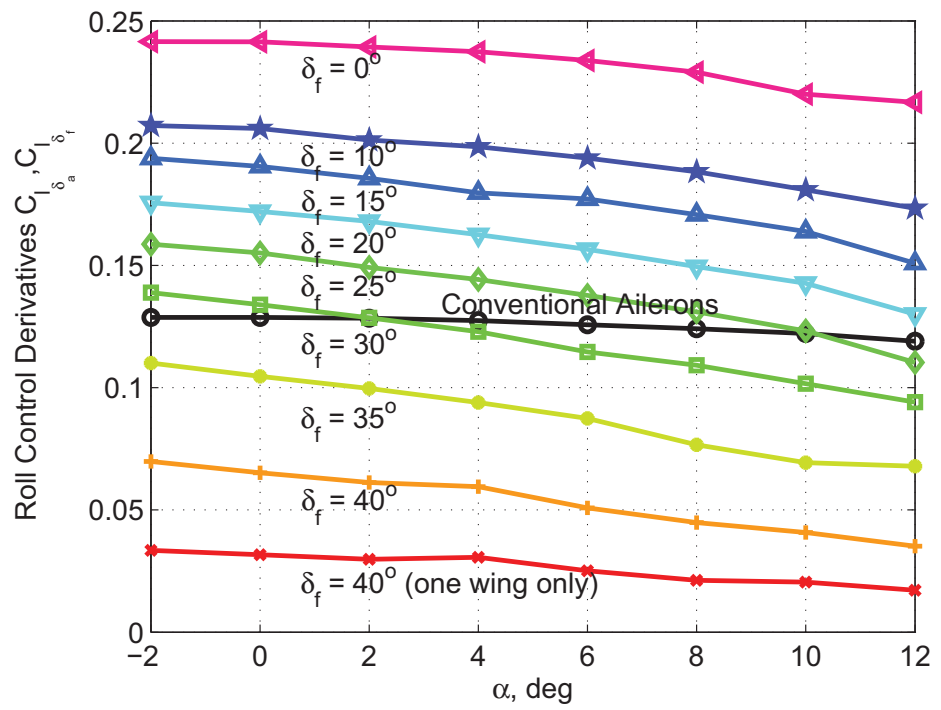

Figure 14. Roll Control Derivatives due to VCCTEF $C_{l_{\delta_{f}}}$ at Various Trim Deflections at Take-Off

The results show that at values where the VCCTEF flap setting has retracted to about $30^{\circ}-25^{\circ}$, a differential deflection of the roll segments of the VCCTEF appear to provide enough roll control derivatives on the order of those of the conventional aileron system.

In summary, the ability to use the roll segments of the VCCTEF to conduct roll maneuvers at cruise and takeoff are evaluated by comparing the roll control derivatives with those for conventional ailerons. At cruise, the roll control segments of the VCCTEF provide greater roll control derivatives than those for the conventional ailerons. At take-off, there exists a threshold at which the VCCTEF has retreated enough such that a differential deflection of the roll control segments of the VCCTEF on both wings can be allowed to provide similar roll control authority to that of a conventional aileron system. For an estimated speed of 150 knots, this threshold occurs when the VCCTEF has retracted to about $30^{\circ}-25^{\circ}$.

\section{Hinge Moment Analysis}

In order to size the VCCTEF actuation, a hinge moment analysis is conducted for the VCCTEF. The hinge moment contributions are due to both the steady-state loads of the aircraft at trimmed flight conditions as well as the maneuver loads. The maneuver loads are established at the maximum design load factors for the aircraft which typically range from - 1 to 2.5 for commercial transport. For commercial transport, the maximum positive load factor is established by FAR 25 "Airworthiness Standards: Transport Category Airplanes" certification according to ${ }^{6}$

$$
n_{\text {pos }} \geq 2.1+\frac{24,000}{W+24,000}
$$


where $W$ is the aircraft weight.

Thus, for a 200,000-lb aircraft, the maximum positive load factor $n_{p o s}$ is established to be greater than +2.2143 . For the scope of this analysis, the maximum positive load factor is thus chosen to be +2.5 . At lower Mach numbers, the maximum load factor is established at the stall angle of attack $\alpha_{\text {stall }}$. At higher Mach numbers, the maximum load factor is limited by structural design limit established by $n_{p o s}$. In analyzing the worst-case hinge moments, coordinated turn maneuvers are assumed to correspond to the maximum load factor.

To conduct a coordinated turn maneuver, a heading angle control is designed to track a coordinated turn command that involves both yaw rate and bank angle command. The linearized heading angle equation obtained by linearization of the kinematic equation is given by

$$
\dot{\psi}=r \sec \bar{\theta}
$$

For small angles, $\dot{\psi}=r$.

When turning, the heading angle as well as the bank angle are changing. A steady-state coordinated turn in the horizontal plane is described by a motion at a constant heading angle rate or yaw rate while the airspeed and altitude are not changing. Thus, the commands for a steady-state coordinated turn are given by

$$
\begin{gathered}
r_{c}=\frac{g \sqrt{n^{2}-1}}{\bar{V}} \\
\phi_{c}=\cos ^{-1} \frac{1}{n}
\end{gathered}
$$

where $n=L / W$ is the load factor.

The lateral-directional dynamics are described by

$$
\left[\begin{array}{c}
\dot{\beta} \\
\dot{p} \\
\dot{r} \\
\dot{\phi}
\end{array}\right]=\left[\begin{array}{cccc}
\frac{Y_{\beta}}{\bar{u}} & \frac{Y_{p}}{\bar{u}}+\bar{\alpha} & \frac{Y_{r}}{\bar{u}}-1 & \frac{g}{\bar{u}} \\
L_{\beta} & L_{p} & L_{r} & 0 \\
N_{\beta} & N_{p} & N_{r} & 0 \\
0 & 1 & 0 & 0
\end{array}\right]\left[\begin{array}{c}
\beta \\
p \\
r \\
\phi
\end{array}\right]+\left[\begin{array}{cc}
\frac{Y_{\delta_{a}}}{\bar{u}} & \frac{Y_{\delta_{r}}}{\bar{u}} \\
L_{\delta_{a}} & L_{\delta_{r}} \\
N_{\delta_{a}} & N_{\delta_{r}} \\
0 & 0
\end{array}\right]\left[\begin{array}{c}
\delta_{a} \\
\delta_{r}
\end{array}\right]
$$

The steady state analysis is governed by

$$
\begin{gathered}
\dot{\phi}=p=0 \\
\dot{\beta}=\frac{Y_{\beta}}{\bar{u}} \beta+\left(\frac{Y_{r}}{\bar{u}}-1\right) r_{c}+\frac{g}{\bar{u}} \phi_{c}+\frac{Y_{\delta_{a}}}{\bar{u}} \delta_{a}+\frac{Y_{\delta_{r}}}{\bar{u}} \delta_{r}=0 \\
\dot{p}=L_{\beta} \beta+L_{r} r_{c}+L_{\delta_{a}} \delta_{a}+L_{\delta_{r}} \delta_{r}=0 \\
\dot{r}=N_{\beta} \beta+N_{r} r_{c}+N_{\delta_{a}} \delta_{a}+N_{\delta_{r}} \delta_{r}=0
\end{gathered}
$$

Solving for $\Delta \beta$ from (18) yields

$$
\beta=-\frac{\left(Y_{r}-\bar{u}\right) r_{c}+g \phi_{c}+Y_{\delta_{a}} \delta_{a}+Y_{\delta_{r}} \delta_{r}}{Y_{\beta}}
$$

Substituting this into (20) and (21) gives the steady state roll and yaw equations

$$
\begin{aligned}
& -\frac{L_{\beta} g}{Y_{\beta}} \phi_{c}+\left[L_{r}-\frac{L_{\beta}\left(Y_{r}-\bar{u}\right)}{Y_{\beta}}\right] r_{c}+\left(L_{\delta_{a}}-\frac{L_{\beta} Y_{\delta_{a}}}{Y_{\beta}}\right) \delta_{a}+\left(L_{\delta_{r}}-\frac{L_{\beta} Y_{\delta_{r}}}{Y_{\beta}}\right) \delta_{r}=0 \\
& -\frac{N_{\beta} g}{Y_{\beta}} \phi_{c}+\left[N_{r}-\frac{N_{\beta}\left(Y_{r}-\bar{u}\right)}{Y_{\beta}}\right] r_{c}+\left(N_{\delta_{a}}-\frac{N_{\beta} Y_{\delta_{a}}}{Y_{\beta}}\right) \delta_{a}+\left(N_{\delta_{r}}-\frac{N_{\beta} Y_{\delta_{r}}}{Y_{\beta}}\right) \delta_{r}=0
\end{aligned}
$$

which can be solved for $\delta_{a}$ and $\delta_{r}$ given $r_{c}$ and $\phi_{c}$ as a function of the load factor $n$.

Since $C_{Y_{\delta_{a}}} \approx 0, C_{n_{\delta_{a}}} \approx 0$, then $\delta_{a}$ and $\delta_{r}$ can be obtained from

$$
\begin{gathered}
-L_{\beta} g \phi_{c}+\left[L_{r} Y_{\beta}-L_{\beta}\left(Y_{r}-\bar{u}\right)\right] r_{c}+L_{\delta_{a}} Y_{\beta} \delta_{a}+\left(L_{\delta_{r}} Y_{\beta}-L_{\beta} Y_{\delta_{r}}\right) \delta_{r}=0 \\
-N_{\beta} g \phi_{c}+\left[N_{r} Y_{\beta}-N_{\beta}\left(Y_{r}-\bar{u}\right)\right] r_{c}+\left(N_{\delta_{r}} Y_{\beta}-N_{\beta} Y_{\delta_{r}}\right) \delta_{r}=0
\end{gathered}
$$

For the cruise condition with $80 \%$ fuel remaining at Mach 0.8 and $30,000 \mathrm{ft}$ corresponding to $\alpha=1.81^{\circ}$ and $\bar{u}=795.6 \mathrm{ft} / \mathrm{sec}$, the stability and control derivatives are given by $C_{Y_{\beta}}=-1.3425, C_{Y_{r}}=1.0552, C_{Y_{\delta_{r}}}=0.4249$, $C_{l_{\beta}}=-0.2444, C_{l_{r}}=0.1743, C_{l_{\delta_{a}}}=0.3332, C_{l_{\delta_{r}}}=0.0533, C_{n_{\beta}}=0.3053, C_{n_{r}}=-0.5307, C_{n_{\delta_{r}}}=-0.2402$. The aircraft properties are specified as $S=1951 \mathrm{ft}^{2}, m=190,000 / 32.174$ slug, $I_{x x}=1,770,000$ slug- $\mathrm{ft}^{2}, I_{z z}=7,270,000$ slug- $\mathrm{ft}^{2}{ }^{5}$ Then 


\begin{tabular}{|c|c|c|}
\hline$Y_{\beta}=\frac{C_{y_{\beta}} \bar{q} S}{m}=-124.8779$ & $L_{\beta}=\frac{C_{l_{\beta}} \bar{q} S b}{I_{x x}}=-9.4684$ & $N_{\beta}=\frac{C_{n_{\beta}} \bar{q} S b}{I_{z z}}=2.8797$ \\
\hline$Y_{r}=\frac{C_{y_{r}} \bar{q} S b}{2 m \bar{u}}=7.7001$ & $L_{r}=\frac{C_{l_{r}} \bar{q} S b^{2}}{2 I_{x x} \bar{u}}=0.5297$ & $N_{r}=\frac{C_{n r} \bar{q} S b^{2}}{2 I_{z z} \bar{u}}=-0.3927$ \\
\hline$Y_{\delta_{a}}=\frac{C_{y_{\delta_{a}}} \bar{q} S}{m} \approx 0$ & $L_{\delta_{a}}=\frac{C_{l_{l_{\sigma}}} \bar{q} S b}{I_{x x}}=12.9087$ & $N_{\delta_{a}}=\frac{C_{n_{\delta_{a}}} \bar{q} S b}{I_{z z}} \approx 0$ \\
\hline$Y_{\delta_{r}}=\frac{C_{y_{\delta_{r}}} \bar{q} S}{m}=39.5237$ & $L_{\delta_{r}}=\frac{C_{l_{\delta_{r}}} \overline{\bar{q}} S b}{I_{x x}}=2.0649$ & $N_{\delta_{r}}=\frac{C_{n_{\delta_{r}}} \bar{q} S b}{I_{z z}}=-2.2656$ \\
\hline
\end{tabular}

Recognizing that $\delta_{a}$ actually represents $\Delta \delta_{f_{3}}$, the differential deflection of the roll control segments of the VCCTEF, a vortex-lattice analysis is conducted to analyze the hinge moments of the aircraft due a differential deflection $\Delta \delta_{f_{3}}$ for maneuver loads and a trim deflection $\left(\delta_{f_{1}}, \delta_{f_{2}}, \delta_{f_{3}}\right)$ of the VCCTEF.

Using pressure data obtained from vortex-lattice method, hinge moments can be obtained for various flight conditions by integrating the pressure distribution aft of the hinge locations. Let $x_{c}$ be the chord line coordinate axis of the clean airfoil section while $x_{c}^{\prime}$ be the chord line axis with the VCCTEF deflected.

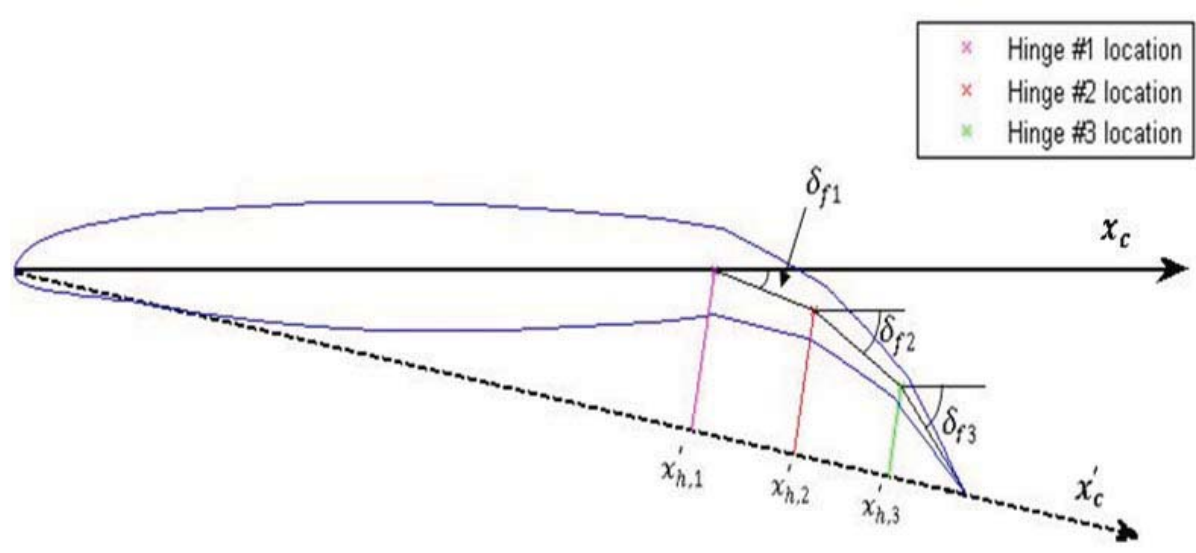

Figure 15. Hinge Moment Locations Along Chord Line

Then, the hinge moment can be obtained by integrating the pressure distribution from the hinge location to the trailing edge as

$$
c_{h_{i}}=\int_{\frac{x_{h_{i}}^{\prime}}{c^{\prime}}}^{1} \Delta c_{p}\left(x_{c}^{\prime}\right)\left(\frac{x_{c}^{\prime}}{c^{\prime}}-\frac{x_{h_{i}}^{\prime}}{c^{\prime}}\right) d\left(\frac{x_{c}^{\prime}}{c^{\prime}}\right)
$$

where $\Delta c_{p}\left(x_{c}^{\prime}\right)=c_{p_{\text {lower }}}\left(x_{c}^{\prime}\right)-c_{p_{\text {upper }}}\left(x_{c}^{\prime}\right)$ is the difference in the pressure coefficient between the lower and upper surfaces at a spanwise wing section, $c^{\prime}$ is the section chord with the VCCTEF deflected, and $x_{h_{i}}^{\prime}$ is the hinge location of the $i$-th hinge line.

The hinge moments are analyzed at two different points in the flight envelope: cruise and take-off. For cruise conditions, a Mach number of 0.8 is assumed at an altitude of 30,000 ft. For an $80 \%$ fuel case, the aircraft weight is assumed to be $190,000 \mathrm{lbs}$ at an angle of attack of $\alpha=1.810^{\circ}$. The hinge moments are calculated with $\delta_{f_{3}}=-14.86^{\circ}$ and $\delta_{r}=-36.38^{\circ}$ corresponding to a load factor of 2.5

To estimate the hinge moments at take-off, the flight condition were chosen to be sea-level flight at Mach 0.228 with a VCCTEF deflection of $\left(\delta_{f_{1}}=10^{\circ}, \delta_{f_{2}}=20^{\circ}, \delta_{f_{1}}=30^{\circ}\right)$. The angle of attack is set at $\alpha=2.434^{\circ}$. This corresponds to the take-off flight condition determined earlier in the roll control analysis to be the threshold at which the VCCTEF is able to provide a sufficient roll control authority similar to that of a conventional aileron system. The maximum load factor of the aircraft is determined by $C_{L_{\max }}$ at $\alpha_{\text {stall }}$. Using a value of $\alpha_{\text {stall }}=10^{\circ}$, a sweep is conducted by calculating the hinge moments for coordinate turn maneuvers corresponding to a range of angle of attack from $\alpha_{\min }=3^{\circ}$ to $\alpha_{\text {stall }}=10^{\circ}$. It is also observed during the sweep that hinge moments could increase on either the right wing with a positive deflection or the left wing with a negative deflection. 


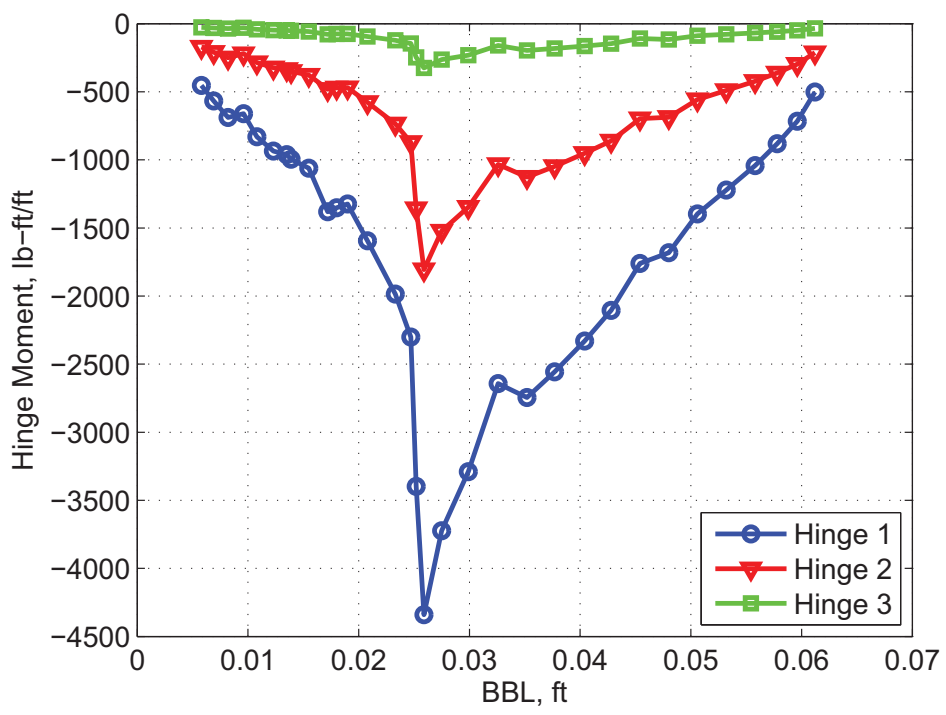

Figure 16. Estimated Worst-Case Hinge Moments

The estimated worst-case hinge moments for the three camber flap segments are determined by combining the largest moments for the cruise and take-off conditions. It is of interest to note that the worst-case hinge moments for the wing portion inboard of the engines are dominated by the hinge moments during take-off with maneuver loads. The worst-case hinge moments on the outboard wing portion are determined by the cruise condition with maneuver loads.

\section{Aeroelastic Modeling and Analysis}

The aeroelastic model of the GTM with VCCTEF is based on one-dimensional structural dynamic theory that captures the aeroelastic deformation of a wing structure in a combined motion that involves flapwise bending, chordwise bending, and torsion. The model includes the effect of aircraft propulsion due to wing flexibility which causes the propulsive forces and moments to couple with the wing elastic motion. Engine mass is also accounted in the model. A fuel management model is developed to describe the wing mass change due to fuel usage in the main tank and wing tanks during cruise.

The model computes both static and dynamic responses of the wing structures. The static aeroelastic deflections are used to estimate the effect of wing flexibility on induced drag and the potential drag reduction by the VCCTEF system. A flutter analysis is conducted to estimate the flutter speed boundary.

The aeroelastic angle of attack includes the rigid-body angle of attack, wing wash-out pre-twist, induced angle of attack due to local downwash, and wing twist and bending deflection. Neglecting the induced angle of attack, the aeroelastic angle of attack at the aerodynamic center of a wing section is given by ${ }^{7}$

$$
\alpha_{a c}=\frac{\alpha}{\cos \Lambda}-\gamma-\Theta+\frac{e \Theta_{t}}{V_{\infty} \cos \Lambda}-W_{x} \tan \Lambda-\frac{W_{t}}{V_{\infty} \cos \Lambda}
$$

where $\gamma$ is the wing wash-out pre-wist angle, $e$ is the forward distance of the aerodynamic center from the elastic center, $V_{\infty}$ is the airspeed, and the subscripts $x$ and $t$ denote the partial derivatives with respect to the wing position $x$ and time $t$.

The aeroelastic angle of attack at the mid-chord location is given by

$$
\alpha_{m c}=\frac{\alpha}{\cos \Lambda}-\gamma-\Theta-\frac{e_{m} \Theta_{t}}{V_{\infty} \cos \Lambda}-W_{x} \tan \Lambda-\frac{W_{t}}{V_{\infty} \cos \Lambda}
$$

where $e_{m}$ is the forward distance of the aeroelastic center from the mid-chord location.

Based on unsteady aerodynamics theory, ${ }^{8}$ the wing section lift coefficient is given by

$$
c_{L_{a c}}=c_{L_{\alpha}} C(k) \alpha_{a c}+c_{L_{\delta}} \delta
$$


where $k=\frac{\omega c}{2 V_{\infty}}$ is the reduced frequency parameter, $\omega$ is the frequency of wing oscillations, $c$ is the section chord, $c_{L_{\alpha}}$ is the section lift curve slope, $c_{L_{\delta}}$ is the section lift derivative, and $\delta$ is the control surface deflection.

The function $C(k)$ is the Theodorsen's complex-valued function which is also expressed in terms of Bessel functions as ${ }^{8}$

$$
C(k)=F(k)+i G(k)
$$

where $F(k)>0$ and $G(k)<0$.

Consider the case when the wing undergoes a simple harmonic motion

$$
\alpha_{a c}=\bar{\alpha} e^{i \omega t}
$$

Then

$$
\dot{\alpha}_{a c}=i \omega \bar{\alpha} e^{i \omega t}=i \omega \alpha_{a c}
$$

Thus the wing section lift coefficient due to unsteady aerodynamics is expressed as

$$
c_{L_{a c}}=c_{L_{\alpha}} \alpha_{a c} F(k)+c_{L_{\alpha}} \frac{\dot{\alpha}_{a c} c}{2 V_{\infty}} \frac{G(k)}{k}+c_{L_{\delta}} \delta
$$

In addition, the apparent mass of the air contributes to the lift force acting at the mid-chord location as

$$
c_{L_{m c}}=\frac{\pi \dot{\alpha}_{m c} c}{2 V_{\infty}}
$$

The total section lift coefficient is

$$
c_{L}=c_{L_{a c}}+c_{L_{m c}}
$$

The section pitching moment coefficient is evaluated as

$$
c_{m}=c_{m_{a c}}+\frac{e}{c} c_{L_{a c}}-\frac{e_{m}}{c} c_{L_{m c}}+c_{m_{\delta}} \delta
$$

where $c_{m_{a c}}$ is the section pitching moment coefficient at the aerodynamic center and $c_{m_{\delta}}$ is the section pitching moment derivative at the aerodynamic center due to the control surface deflection $\delta$.

The combined bending and torsion aeroelastic equations are given by

$$
\begin{aligned}
\left(G J \Theta_{x}\right)_{x}= & {\left[c c_{m_{a c}}+e c_{L_{\alpha}}\left(\frac{\alpha}{\cos \Lambda}-\gamma-\Theta+\frac{e \Theta_{t}}{V_{\infty} \cos \Lambda}-W_{x} \tan \Lambda-\frac{W_{t}}{V_{\infty} \cos \Lambda}\right) F(k)\right.} \\
& +e c_{L_{\alpha}}\left(\frac{\dot{\alpha}}{\cos \Lambda}-\Theta_{t}+\frac{e \Theta_{t t}}{V_{\infty} \cos \Lambda}-W_{x t} \tan \Lambda-\frac{W_{t t}}{V_{\infty} \cos \Lambda}\right) \frac{c}{2 V_{\infty}} \frac{G(k)}{k}+\left(c c_{m_{\delta}}+e c_{L_{\delta}}\right) \delta \\
\left.-e_{m} \frac{\pi c}{2 V_{\infty}}\left(\frac{\dot{\alpha}}{\cos \Lambda}-\Theta_{t}-\frac{e_{m} \Theta_{t t}}{V_{\infty} \cos \Lambda}-W_{x t} \tan \Lambda-\frac{W_{t t}}{V_{\infty} \cos \Lambda}\right)\right] q_{\infty} \cos ^{2} \Lambda c-m g e_{c g}+m k^{2} \Theta_{t t}-m e_{c g} W_{t t} & +\delta\left(x-x_{e}\right)\left(I_{e} \Theta_{t t}-m_{e} y_{e} W_{t t}-m_{e} g y_{e}\right) \\
\left(E I W_{x x}\right)_{x x}= & {\left[c_{L_{\alpha}}\left(\frac{\alpha}{\cos \Lambda}-\gamma-\Theta+\frac{e \Theta_{t}}{V_{\infty} \cos \Lambda}-W_{x} \tan \Lambda-\frac{W_{t}}{V_{\infty} \cos \Lambda}\right) F(k)\right.} \\
& \quad+c_{L_{\alpha}}\left(\frac{\dot{\alpha}}{\cos \Lambda}-\Theta_{t}+\frac{e \Theta_{t t}}{V_{\infty} \cos \Lambda}-W_{x t} \tan \Lambda-\frac{W_{t t}}{V_{\infty} \cos \Lambda}\right) \frac{c}{2 V_{\infty}} \frac{G(k)}{k}+c_{L_{\delta}} \delta \\
+ & \left.\frac{\pi c}{2 V_{\infty}}\left(\frac{\dot{\alpha}}{\cos \Lambda}-\Theta_{t}-\frac{e_{m} \Theta_{t t}}{V_{\infty} \cos \Lambda}-W_{x t} \tan \Lambda-\frac{W_{t t}}{V_{\infty} \cos \Lambda}\right)\right] q_{\infty} \cos { }^{2} \Lambda c-m g-m W_{t t}+m e_{c g} \Theta_{t t} \\
& -\delta\left(x-x_{e}\right)\left(m_{e} W_{t t}-m_{e} y_{e} \Theta_{t t}+m_{e} g\right)
\end{aligned}
$$

where $E I$ is the bending rigidity, $G J$ is the torsional rigidity, $q_{\infty}$ is the dynamic pressure, $m$ is the wing mass per unit length, $e_{c g}$ is the forward distance of the center of mass from the elastic center, $k$ is the section radius of gyration, $I_{e}$ 
is the engine rotary mass inertia, $m_{e}$ is the engine mass, $y_{e}$ is engine offset from the elastic axis, and $\delta\left(x-x_{e}\right)$ is the Dirac delta function to model the point mass of the engine.

The aeroelastic equations are solved using the finite-element method. A computer code is developed in Matlab to model the wing aeroelasticity. The model is coupled with vortex-lattice code Vorview, which provides the aerodynamic information, to perform a flutter analysis and compute static aeroelastic deflections of a wing structure.

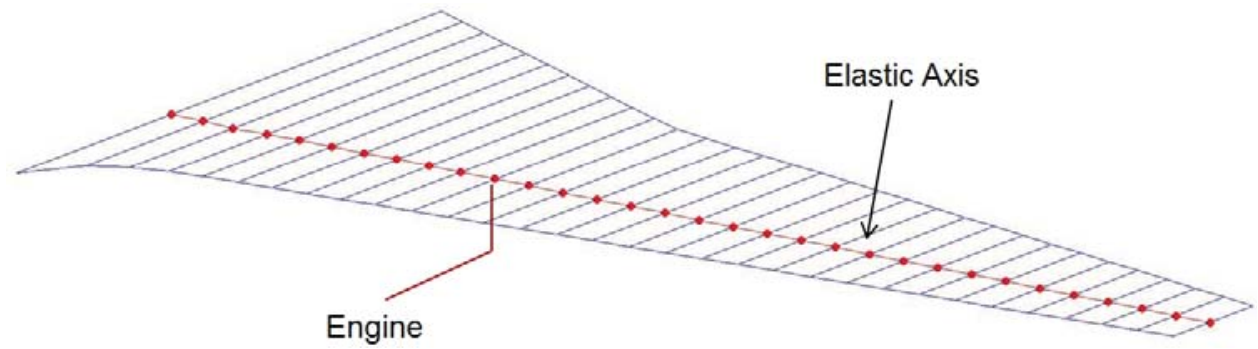

Figure 17. Wing Finite-Element Model

To model wing aeroelasticity, the values of structural rigidities $E I$ and $G J$ are estimated for a conventional stiff wing structure. To explore the potential drag reduction benefit, a "softened" wing structure is proposed by reducing the wing structural rigidities $E I$ and $G J$ by a factor of 2 to model a highly flexible wing structure. The increased wing flexibility enables the wing shaping control actuation by the VCCTEF system.

To relate the wing deflection at cruise to the wing mass, a fuel mass management is modeled. The fuel for the GTM is stored in the center tank and wing main tanks. The center tank holds 20,000 lbs of fuel. Each of the main tanks holds about 15,000 lbs of fuel. The center tank is used first until it is empty. Then, the fuel is drawn equally from the wing main tanks. The fuel mass is modeled as the combined wing mass density. As the structural rigidities are reduced, the wing dry mass also decreases. Assuming that the wing box structure is modeled as a thin-walled structure, then the mass change is related to the change in the wing structural rigidity EI according to Figure 18.9

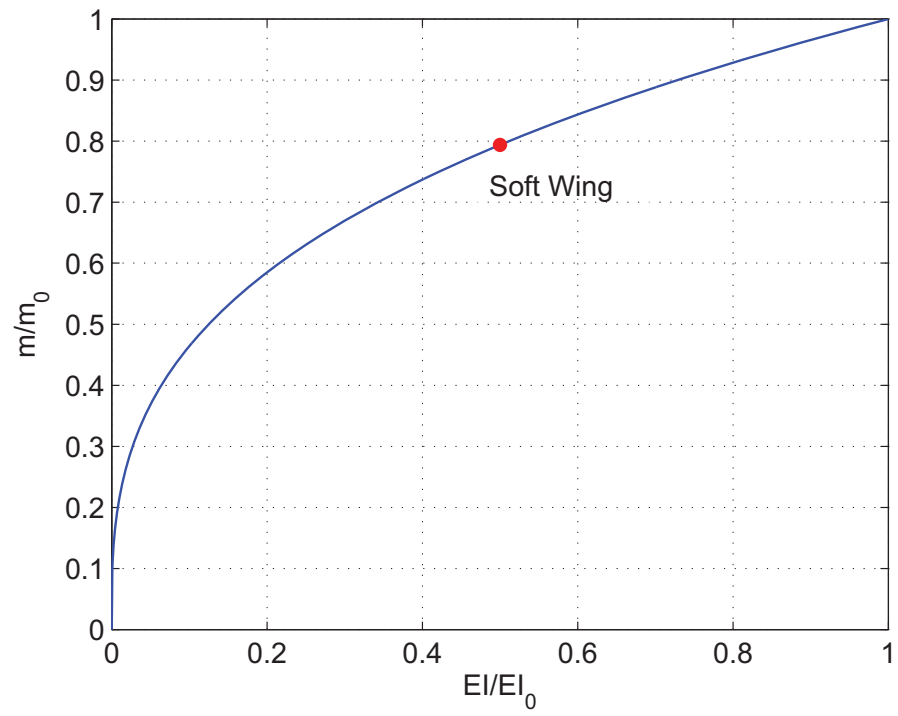

Figure 18. Mass vs. Structural Rigidity

The flutter speed prediction is computed using a linear aeroelastic model with an assumed $1 \%$ damping. The frequencies and damping ratios of the first four symmetric and anti-symmetric modes for the stiff wings and softened wings are plotted in Figures 19 and 20 as a function of the equivalent airspeed in knots. ${ }^{9}$ The critical flutter mode is the second bending anti-symmetric mode. Figure 21 shows the flutter boundaries of the GTM with the stiff wings and softened wings. ${ }^{9}$ The flutter boundary for the GTM with the softened wings appears inside the flight envelope at high altitude. The reduction in the structural rigidities causes a significant drop in the flutter speed. As a result, active aeroservoelastic (ASE) mode suppression control would likely be required. 

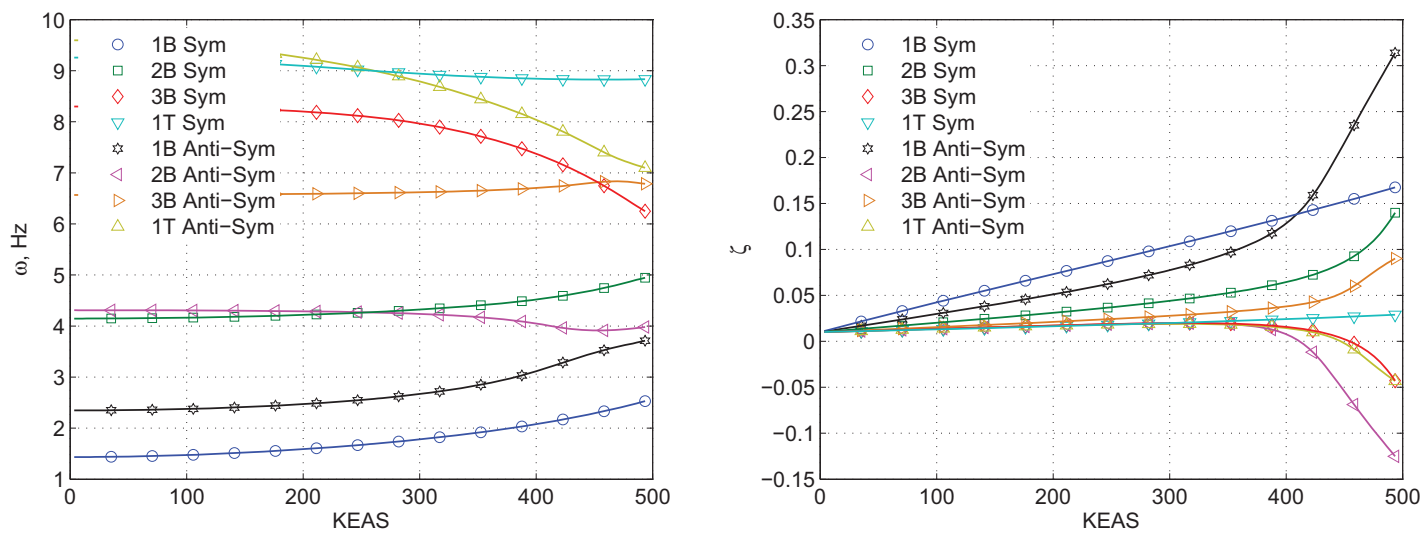

Figure 19. Frequencies and Damping Ratios of GTM with Stiff Wings at 35,000 ft
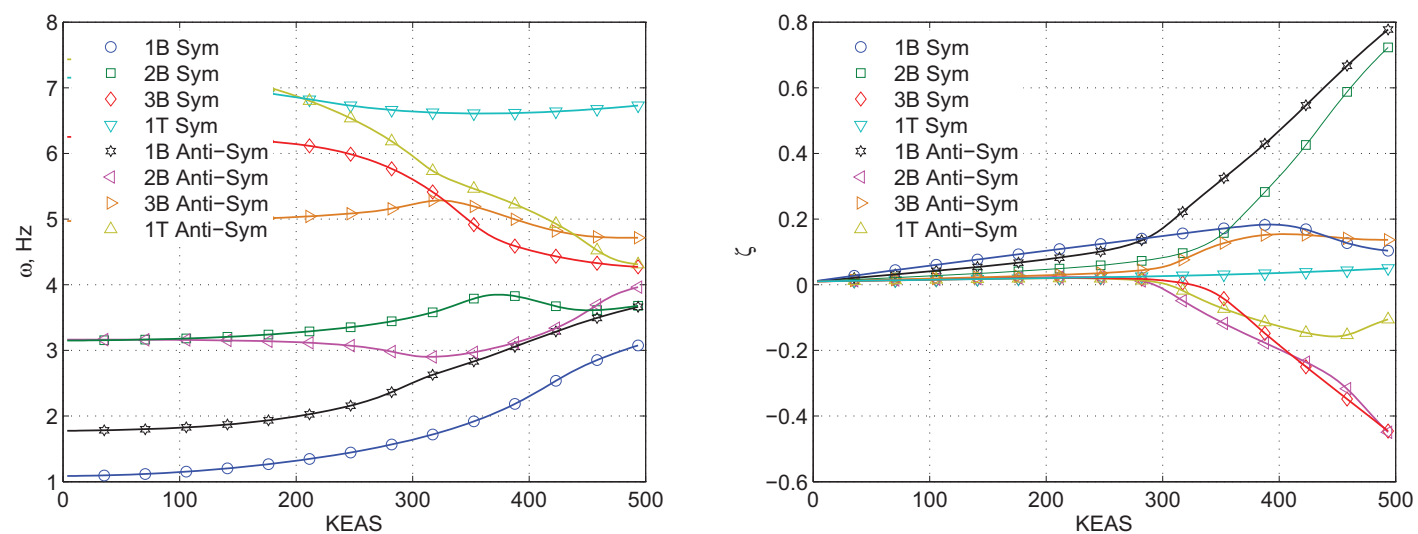

Figure 20. Frequencies and Damping Ratios of GTM with Softened Wings at 35,000 ft

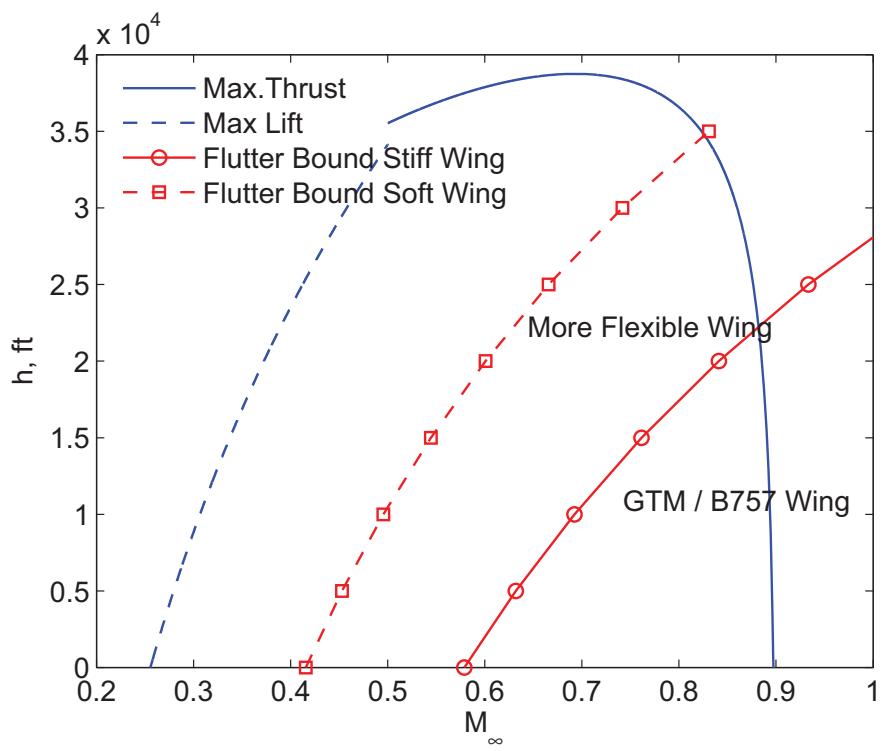

Figure 21. Flight Envelope Flutter Boundary Changes with a More Flexible Wing 


\section{Aerodynamic Modeling}

\section{A. Skin Friction Drag Estimation}

Vortex-lattice code Vorview is used to conduct aerodynamic modeling and analysis. Due to the inherent limitation of the vortex-lattice method, Vorview only computes induced drag component. To analyze the drag reduction benefit, a skin friction drag correction is implemented. Wave drag due to compressibility effect can be analyzed by CFD which will be studied in the future. Assuming the cruise condition is reasonably far from the drag divergence Mach number, then the wave drag component may be neglected in the initial analysis.

Currently, the calculation of viscous drag, using an approximate method to estimate skin friction as described by Abbott and Von Doenhoff, ${ }^{10}$ in the process of being added to Vorview. This section describes the method, as well as correlation of preliminary results with previously published data.

The following relationships form the basis for approximating viscous drag:

$$
\begin{gathered}
C_{D_{f, \text { wing }}}=k c_{f} \frac{S_{w_{\text {wing }}}}{S_{\text {ref }}} \\
C_{D_{f, \text { fuselage }}}=K c_{f} \frac{S_{w_{\text {fuselage }}}}{S_{\text {ref }}}
\end{gathered}
$$

The key parameters in these expressions are the calculation of skin friction coefficient, $c_{f}$, and the corresponding form factors for airfoil, $k$, and fuselage, $K$, shapes. The airfoil form factor $k$ is given by Reference ${ }^{11}$ as a function of the thickness-to-chord ratio $t / c$, Mach number $M$, and wing sweep angle $\Lambda$. The fuselage (body) form factor $K$ is given by Reference ${ }^{12}$ as a function of the fineness ratio $l / d$ and Mach number $M$.

The calculation of skin friction coefficient associated with the wing and fuselage is performed using one side of a flat plate approximation. The resulting expression for $c_{f}$ that captures the effect of laminar-to-turbulent flow transition is as follows:

$$
c_{f}=\frac{X_{c}}{\bar{c}}\left(1.328 R e_{X_{c}}^{-0.5}\right)+\left(0.072 R e_{\bar{c}}^{-0.2}\right)-\frac{X_{c}}{\bar{c}}\left(0.072 R e_{X_{c}}^{-0.2}\right)
$$

where $R e_{\bar{c}}$ is the Reynolds number based on the mean aerodynamic chord.

To estimate the transition length, $X_{c}$, which is the distance from the leading edge of the wing or nose of the fuselage where the flow transitions from laminar to turbulent flow, the following expression is used:

$$
X_{c}=\frac{\operatorname{Re}_{X_{c}} \mu}{\rho V}
$$

where the value of $\operatorname{Re}_{X_{c}}$ is assumed to be 600,000 for this study, $\mu$ is the kinematic (absolute) viscosity, $\rho$ is the density, and $V$ is the airspeed.

For varying values of $\operatorname{Re}_{X_{c}}$ and airspeed at a given altitude, this expression yields the transition lengths depicted in Figure 22.

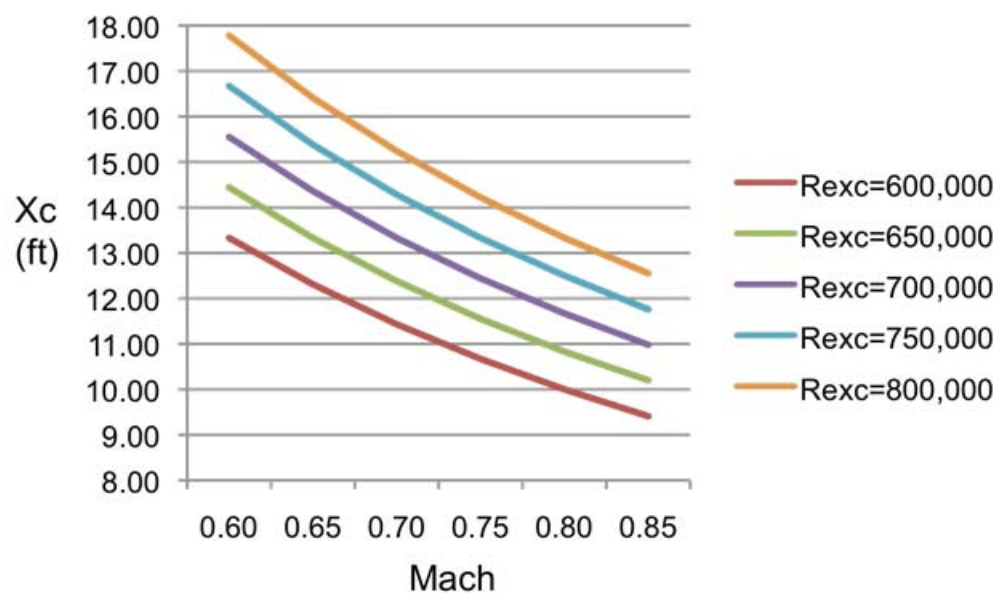

Figure 22. Transition Length Variation at $35 \mathrm{~K} \mathrm{ft}$ altitude (Standard Day) 
As can be seen in Figure 23, vortex-lattice analysis alone underestimates the drag for the DLRF6 reference aircraft. ${ }^{13}$ However, when viscous effects are added to the vortex-lattice drag estimates, the results appear much more reasonable, especially within an angle-of-attack range near the minimum drag point.

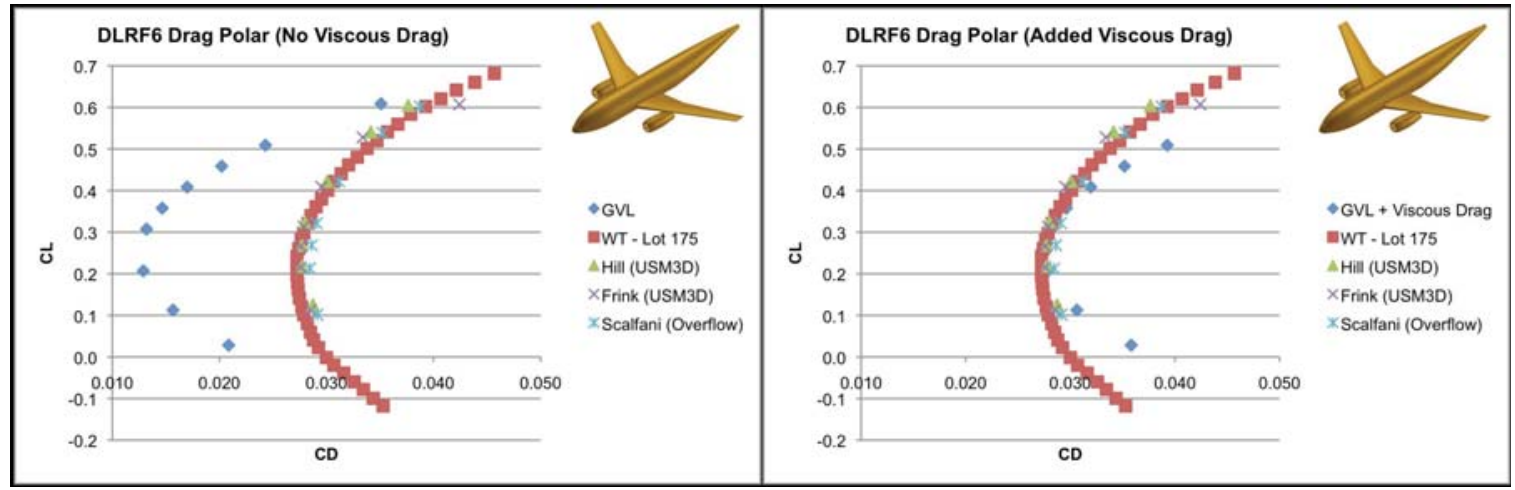

Figure 23. Preliminary Comparison of Drag Estimates with Previously Published Data for the DLRF6 Reference Aircraft

\section{B. High Lift Estimation}

To estimate high-lift capabilities of the VCCTEF for take-off and landing, an initial assessment is conducted using a vortex-lattice analysis. Assuming there is no significant flow separation due to the camber surface of the VCCTEF, a vortex-lattice analysis could give a first-order approximation of high-lift capabilities. A series of Vorview runs is conducted for various circular-arc camber settings for the VCCTEF up to a commanded flap deflection $\delta_{f}=40^{\circ}$. To estimate the value of $C_{L_{\max }}$, a stall angle of attack $\alpha_{\text {stall }}$ must be known. Wind tunnel data for the GTM acquired in the 14-Foot-By-22-Foot Wind Tunnel at NASA Langley Research Center ${ }^{14}$ are available for use to determine the value of $\alpha_{\text {stall }}$. Previous vortex-lattice Vorview data ${ }^{5}$ and Cart $3 d$ Euler CFD data ${ }^{15}$ were computed for the same wind tunnel runs to compare the results with the wind tunnel data as shown in Figure 24.

The vortex-lattice data are generally in an excellent agreement with the wind tunnel data up to $\alpha_{\text {stall }}$ which is estimated to be about $12^{\circ}$. For flaps are deployed, in general, $\alpha_{\text {stall }}$ is less than the value for a clean wing configuration. From Roskam and Lan, ${ }^{6}$ the stall angle of attack with flap deployments is estimated to be reduced by $2^{o}$. This results in $\alpha_{\text {stall }}=10^{\circ}$. The value of $C_{L_{\max }}$ for the VCCTEF can then be estimated.

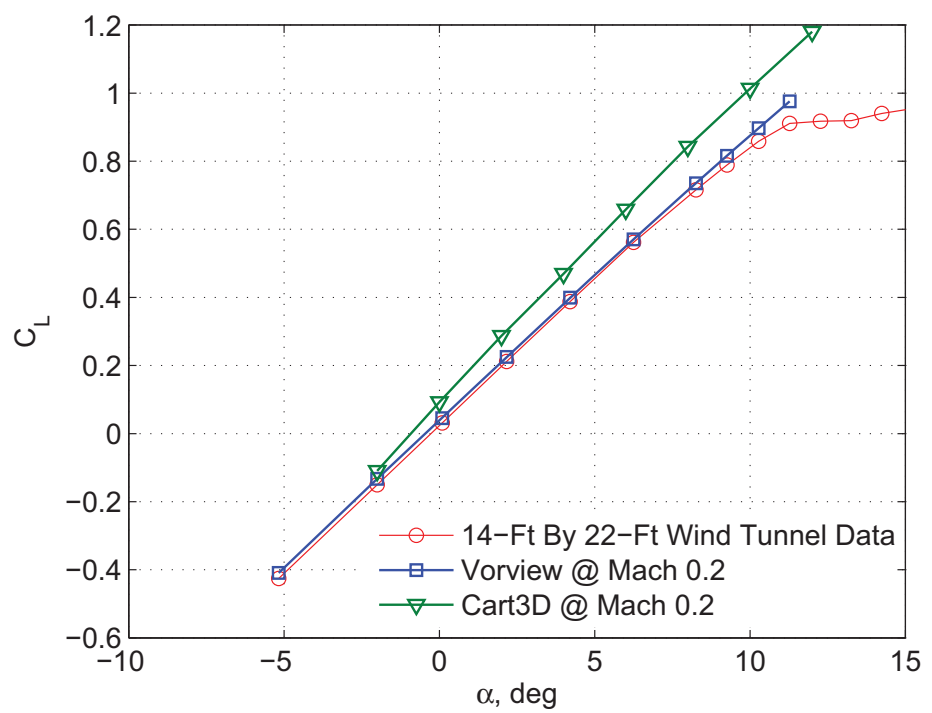

Figure 24. Comparison of Vortex-Lattice and CFD Data with Wind Tunnel Data for GTM

The pressure distributions with the VCCTEF in a high-lift configuration are plotted in Figures 25 and 26. 


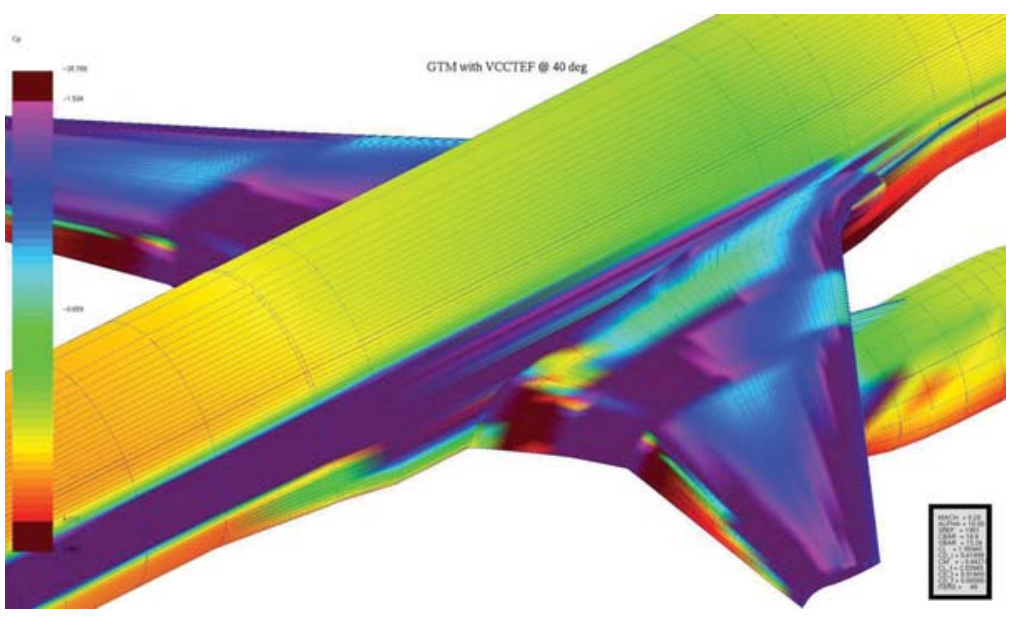

Figure 25. Pressure Distribution on Upper Wing Surface with VCCTEF at $\delta_{f}=40^{\circ}$

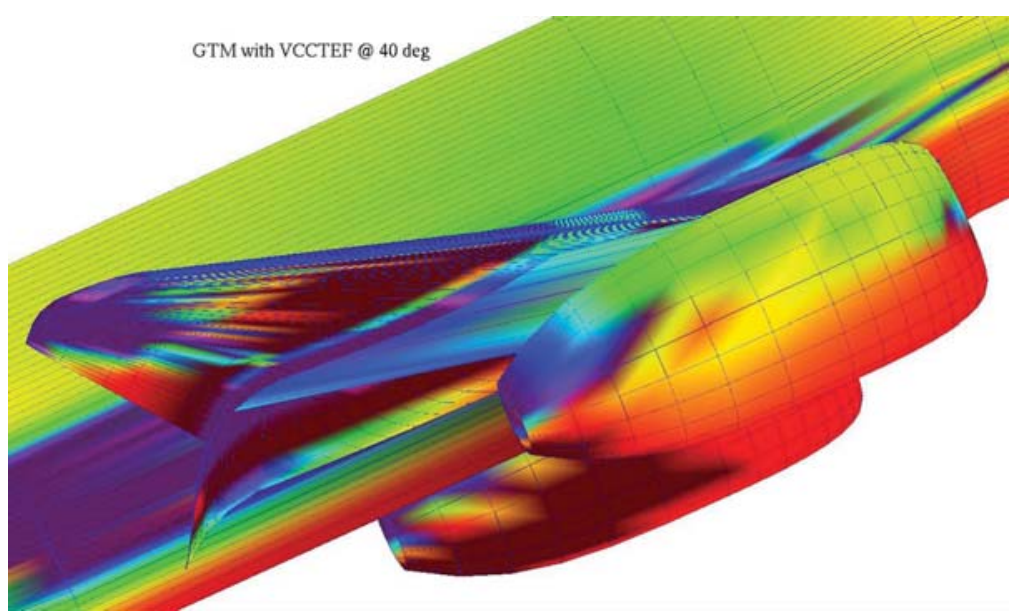

Figure 26. Pressure Distribution on Lower Wing Surface with VCCTEF at $\delta_{f}=40^{\circ}$

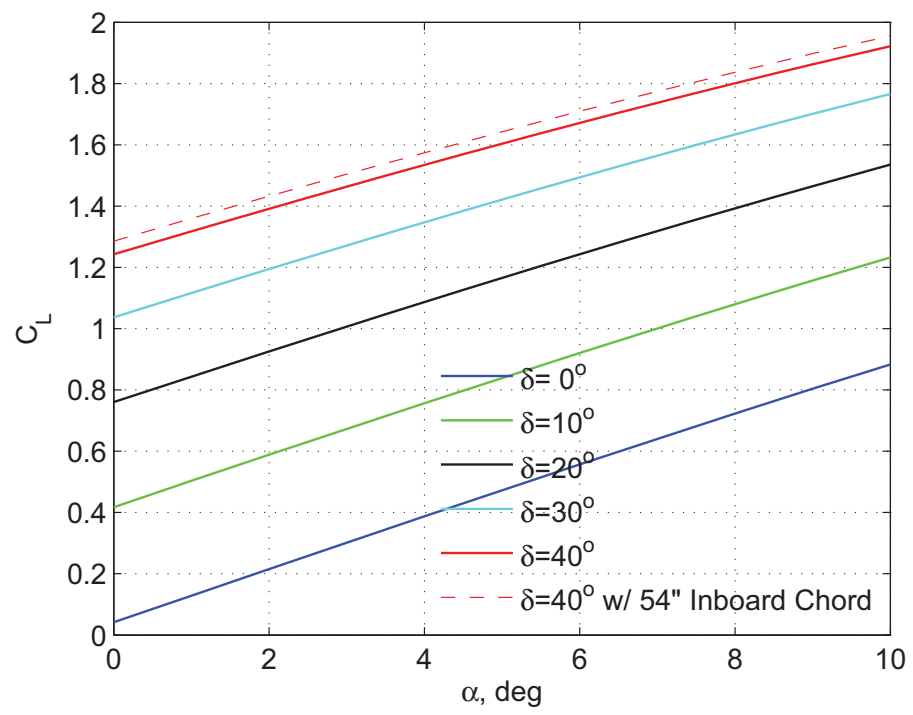

Figure 27. Lift Curves of GTM with VCCTEF 
Figure 27 shows the lift curves for various commanded flap deflections. The VCCTEF chord varies from 24 inches at the wing tip to 48 inches at the junction with the inboard wing portion. At $\delta_{f}=40^{\circ}$, a value of $C_{L_{\max }}=1.921$ is obtained. It is realized that this $C_{L_{\max }}$ may not be enough. As a result, a flap chord of 54 inches instead of 48 inches is proposed. The value of $C_{L_{\max }}$ increases to 1.956 .

To compare the $C_{L_{\max }}$ estimate with that for a conventional flap system, the value of $C_{L_{\max }}$ for a conventional flap system deployed at $40^{\circ}$ is estimated by a method presented in Roskam and Lan. ${ }^{6}$ A value of $C_{L_{\max }}=2.119$ is obtained. With the conventional flap deployed and extended, the wing surface area increases by $10 \%$ for the GTM. This results in a stall speed of $V_{\text {stall }}=114$ knots. Assuming that an approach speed of $23 \%$ greater than the stall speed, an approach speed of $V_{\text {approach }}=140$ knots is estimated. This approach speed is very reasonable for an aircraft of this size.

Without having an airgap, it appears that the VCCTEF may not provide sufficient lift capabilities since the wing surface area is not extended. To achieve the same stall speed of $V_{\text {stall }}=114$ knots without flap extension, the VCCTEF would require to achieve a value of $C_{L_{\max }}=2.330$. From Abbott and Von Doenhoff, a slotted slat and flap configuration contributes about 0.311 to $C_{L_{\max }}$. Thus, a value of $C_{L_{\max }}=2.267$ is estimated for the VCCTEF with a slotted configuration. This $C_{L_{\max }}$ value results in a stall speed of $V_{\text {stall }}=116$ knots.

\section{Drag Optimization}

The VCCTEF has 14 camber spanwise flap sections designed to shape the spanwise wing lift distribution to obtain optimal lift-to-drag ratios throughout the flight envelope. Furthermore, each spanwise flap has three chordwise camber flap segments that can also be used to optimize the chordwise wing lift distribution. The three spanwise constant flap sections of the inboard wing portion can also be used to optimize the spanwise and chordwise wing lift distributions. Thus, there are a total of 45 configuration variables that describe a VCCTEF configuration. As the aircraft cruises, the fuel loading causes a change in the trim lift. In addition, the wing aeroelastic deflections also causes the wing lift distribution to change. Thus, as the fuel loading moves away from the design point which is typically at $50 \%$ fuel loading, the wing aerodynamics can become non-optimal. Wing shaping control using the VCCTEF allows the wing lift distribution to be re-optimized at off-design flight conditions. This feature potentially can have a significant drag reduction benefit.

An initial induced drag optimization is conducted with a circular arc camber VCCTEF using 15 configuration variables; 14 commanded flap deflections for the VCCTEF outboard of the engine, and one commanded flap deflection for the three spanwise constant flap sections inboard from the engine. The initial optimization is conducted with a rigid wing configuration, that is, there is no account for wing aeroelastic deflections. Moreover, the optimization is performed with a jig-shape wing. The optimization is subject to a cruise lift constraint that factors into the fuel loading.

The flexible skin materials that cover the spanwise camber flap sections create constraints to the flap deflections. These constraints impose a certain relative flap deflection between any two adjacent spanwise flap sections. An unconstrained optimization is conducted first. Then, relative deflection constraints of $1^{\circ}$ and $2^{\circ}$ are added to the optimization. The unconstrained optimization results serve as upper limits of drag optimization with relative deflection constraints.

The results of the optimization are shown in Table 2.

\begin{tabular}{|l|c|c|c|}
\hline & Unconstrained & $2^{\circ}$ Constraint & $1^{\circ}$ Constraint \\
\hline \hline $80 \%$ Fuel Loading & $2.64 \%$ & $2.38 \%$ & $1.99 \%$ \\
\hline $50 \%$ Fuel Loading & $3.64 \%$ & $3.26 \%$ & $1.23 \%$ \\
\hline $20 \%$ Fuel Loading & $9.88 \%$ & $5.71 \%$ & $3.24 \%$ \\
\hline
\end{tabular}

Table 2. Preliminary Induced Drag Optimization for Rigid Wing with Circular Arc Camber VCCTEF

The largest drag reduction is noted with $20 \%$ fuel loading. For $2^{\circ}$ relative deflection constraints, the drag reduction decreases to $5.71 \%$ from $9.88 \%$. There is a significant penalty in drag reduction benefit as the relative deflection constraints drop below $2^{\circ}$. This suggests that the flexible skin materials should be designed to allow at least $2^{\circ}$ flexibility.

The optimal VCCTEF configuration for the $20 \%$ fuel loading is shown in Figure 28. 


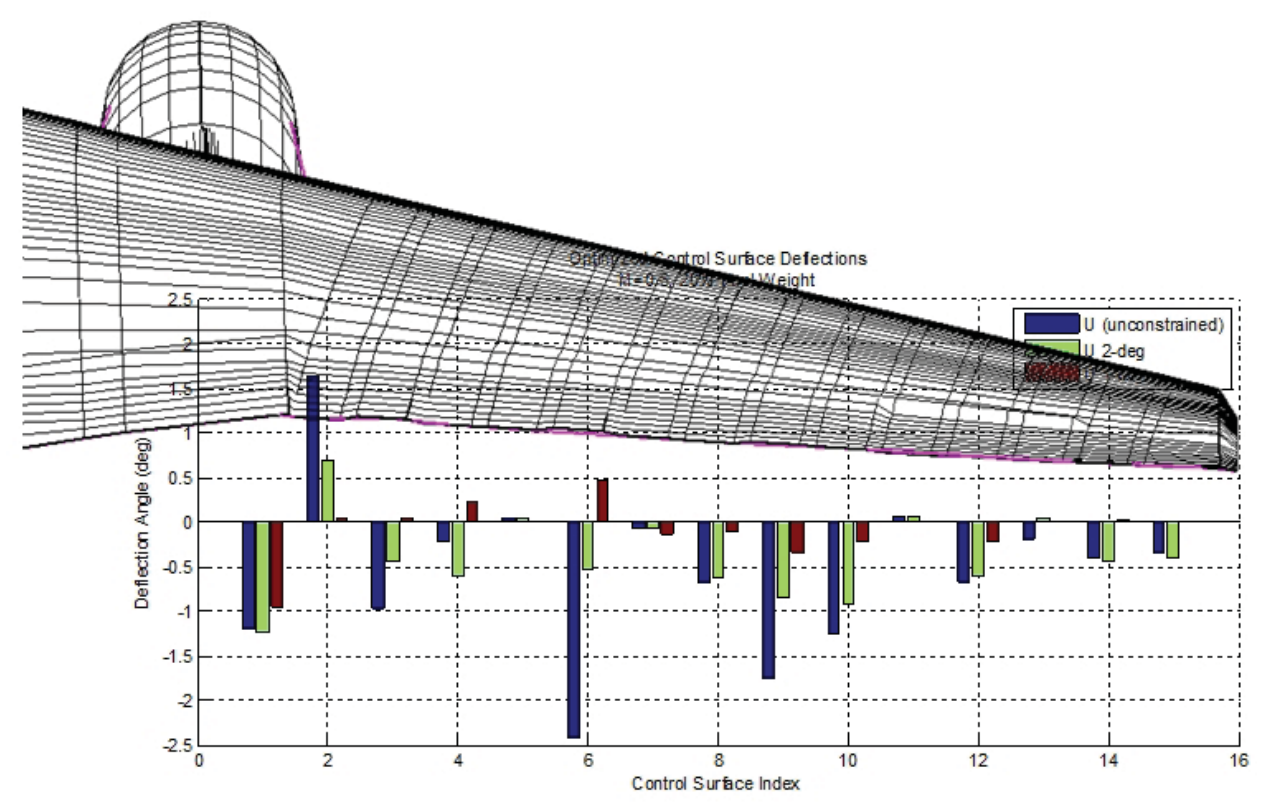

Figure 28. Optimal VCCTEF Configuration for 20\% Fuel Loading

It should be noted that these preliminary results only indicate qualitatively that drag reduction can indeed be achieved with the VCCTEF. Additional optimization work that includes effects of aeroelasticity and additional configuration variables will be conducted in the near future to estimate more precisely the potential drag reduction benefit of the VCCTEF.

\section{Application to Transport Aircraft}

This study shows that to gain the advantages of wing shaping control, configuration changes in high-lift devices have to be part of the wing shaping control strategy. Flap and slat devices inherently generate drag as they increase lift. Conventional flap and slat systems as in the current generation aircraft are not aerodynamically efficient to maximize drag-reducing control strategies like wing shaping control. The variable camber continuous trailing edge flap concept developed in this study does offer a potential pay-off for drag reduction even when used in current generation aircraft. Technical challenges do exist such as the increase in the number of multiple segmented flaps that form a variable camber continuous trailing edge flap surface and can lead to increased design complexity.

The issues of wing flexibility on vehicle stability cannot be ignored. Flight control can be used to stabilize aeroelastic instability of wing modes due to wing flexibility. Aeroelastic tailoring by properly distributing wing stiffness throughout the airframe may also improve stability margins of aeroelastic modes. The role of flight control is to provide this stability augmentation which would reduce the demand on a flight control system. Increased wing flexibility may result in more susceptibility to potentially adverse responses to air turbulence and wing gusts. Flight control design would need to take this issue into consideration. The VCCTEF control capability may be useful for turbulence mitigation. Gust load alleviation control technology has been deployed on modern commercial aircraft. Such technology may become standard one day for aircraft flight control design as the trend in aircraft design is moving toward a more flexible airframe design for increased performance and reduced fuel burn.

Advantages of the Variable Camber Continuous Trailing Edge Flap system include:

1. Cruise drag reduction: Use of deflecting wing control surfaces to achieve drag-reducing wing twist shape raises the question of how much drag is added back due to the flap deflection. The camber design of the flap will reduce some of this flap drag as compared to using conventional flap / aileron control.

2. High-lift configurations that have less drag: The cambered high-lift full span flaps offer less drag than conventional flaps during full or partial flap extension. This can reduce fuel burn during the sometimes long flight segments requiring flaps, such as air traffic flight paths at busy airports.

3. Roll control: Use of the aft camber as a roll control effector may be more effective than outer wing ailerons in control of flexible wing reversal. 
4. Stabilization of wing dynamic modes: The third or aft camber section can be used to suppress wing dynamic modes, having sufficient bandpass for this control. Moreover, the control input can be distributed along the span of the wing, making the suppression more effective.

5. Weight savings for flap actuation components: Two advantages for weight savings with the VCCTEF system are: 1) Shape Memory Alloy actuators have much less weight compared with electric or hydraulic actuation; exceeding better than a 10:1 advantage; and 2) the VCCTEF uses hinge line rotary actuators, thereby eliminating the need for bell-cranks and heavy screw-jack actuators with external mounting and pod covers. This can result in a weight savings of between 250 and 500 lbs. per wing.

\section{Conclusion}

The Variable Camber Continuous Trailing Edge Flap has excellent potential to achieve energy goals for future N+3 transports. The trend for lighter and more flexible wing structures can be leveraged to upgrading structural shape to meet combined aerodynamic and aeroelastic continuous wing shaping for drag reduction. Adding new technology of Shape Memory Alloy actuation results in much lighter weights for flap control components. Factoring in continuous camber flaps eliminates drag-inducing gaps between wing control surfaces, and also can significantly reduce noise due to these gaps.

This combination of aeroelastic and aerodynamic properties that results in performance improvement is a good example of effective Multidisciplinary Design Analysis and Optimization (MDAO) system development. Program results also project the need for verification of interactive aeroelastic and aerodynamic modeling; this being best achieved by wind tunnel tests of flexible wing models with variable camber control surfaces. Flight development on flexible wing test vehicles would further refine and verify the control of the flexible wing, especially control of possible flutter excitations. Boeing and NASA are continuing exploring this VCCTEF concept using a more flexible wing configured in the Generic Transport Model based on the B757, with wing flexible properties closer to current transport (i.e., B787) wing designs.

\section{Acknowledgment}

The authors wish to acknowledge Mr. John Dykman, Mr. Dan Clingman, Mr. Charles Morris, and Mr. Jim Sheahan from Boeing for technical support in design and aeroelastic model development. The authors also want to thank the NASA Aeronautics Research Mission Directorate (ARMD) Fixed Wing Project under the Fundamental Aeronautics Program for providing the funding support for this work.

\section{References}

\footnotetext{
${ }^{1}$ Nguyen, N., "Elastically Shaped Future Air Vehicle Concept," NASA Innovation Fund Award 2010 Report, October 2010, Submitted to NASA Innovative Partnerships Program.

${ }^{2}$ Boeing Report No. 2012X0015, “Development of Variable Camber Continuous Trailing Edge Flap System,” October 4, 2012.

${ }^{3}$ Jordan, T. L., Langford, W. M., Belcastro, C. M., Foster, J. M., Shah, G. H., Howland, G., and Kidd, R., "Development of a Dynamically Scaled Generic Transport Model Testbed for Flight Research Experiments,” AUVSI Unmanned Unlimited, Arlington, VA, 2004.

${ }^{4}$ Miranda, L.R., Elliot, R.D., and Baker, W.M., "A Generalized Vortex Lattice Method for Subsonic and Supersonic Flow Applications," NASA CR-2865, 1977.

${ }^{5}$ Nguyen, N., Nelson, A., and Pulliam, T., "Damage Adaptive Control System Research Report,” Internal NASA Report, April 2006.

${ }^{6}$ Roskam and Lan, Airplane Aerodynamics and Performance, DARcorporation, 1997.

${ }^{7}$ Nguyen, N., Trinh, K., Nguyen, D., Tuzcu, I., "Nonlinear Aeroelasticity of Flexible Wing Structure Coupled with Aircraft Flight Dynamics," AIAA Structures, Structural Dynamics, and Materials Conference, AIAA-2012-1792, April 2012.

${ }^{8}$ Theodorsen, T, "General Theory of Aerodynamic Instability and the Mechanism of Flutter", NACA Report 496, 1935.

${ }^{9}$ Nguyen, N., Urnes, J., "Aeroelastic Modeling of Elastically Shaped Aircraft Concept via Wing Shaping Control for Drag Reduction," AIAA Atmospheric Flight Mechanics Conference, AIAA-2012-4642, August 2012.

${ }^{10}$ Abbott, I. H. and Von Doenhoff, A. E., Theory of Wing Sections, Dover Publications, Inc., New York, pp. 97-103.

${ }^{11}$ Stanford University, AA241, http://adg.stanford.edu/aa241/drag/lsformfactor.html

${ }^{12}$ Stanford University, AA241, http://adg.stanford.edu/aa241/drag/BODYFORMFACTOR.HTML

${ }^{13}$ Hill, G., "Aerodynamic and Acoustic Investigations of an Advanced Over-the-Wing Nacelle Transport Aircraft Configuration," M.S. Thesis, Old Dominion University, May, 2007 (p. 35, Fig. 6).

${ }^{14}$ Shah, G. H., 'Aerodynamic Effects and Modeling of Damage to Transport Aircraft," AIAA Atmospheric Flight Mechanics Conference, AIAA-2008-6203, August 2008.
} 
${ }^{15}$ Aftosmis, M. J., Berger, M. J., and Melton, J. E., "Robust and Efficient CartesianMesh Generation for Component- Based Geometry," AIAA Journal, Vol. 36, No. 6, 1998, pp. 953-960. 